\title{
On multiscale computational mechanics with time-space homogenization
}

\author{
Pierre Ladevèze ${ }^{\mathrm{b}, *}$, David Néron, Jean-Charles Passieux \\ LMT-Cachan \\ (ENS Cachan/CNRS UMR8535/UPMC/PRES UniverSud Paris) \\ 61, avenue du Président Wilson, F-94235 Cachan Cedex, France \\ ${ }^{\mathrm{b}}$ EADS Foundation Chair "Advanced Computational Structural Mechanics"
}

\begin{abstract}
The paper deals with multiple scales in both space and time. First, the state-ofthe-art is presented. Then, we discuss a family of computational approaches using time-space homogenization. Emphasis is put on the time aspects.
\end{abstract}

Key words: multiscale in time and space, homogenization, domain decomposition, LATIN, parallel computing, proper orthogonal decomposition.

\section{Introduction}

Today, in structural mechanics, there is a growing interest in a class of techniques called "multiscale computational approaches", which are capable of analyzing structures in which two or more very different scales can be identified. A typical engineering example is that of a relatively large structure in which local cracking or local buckling occurs $[1,2]$. Another typical engineering problem is related to the increasing interest in material models described on a scale smaller than that of the macroscopic structural level, with applications ranging from the design of composite materials and structures to manufacturing $[3,4]$. In such situations, the structure being studied is highly heterogeneous and the local solution involves short-wavelength phenomena in both space and time. As a result, classical finite element codes lead to systems with very large numbers of degrees of freedom and the corresponding calculation costs are generally prohibitive. Therefore, one of today's main challenges is to

* Corresponding author. E-mail: ladeveze@lmt.ens-cachan.fr 
derive computational strategies capable of solving such engineering problems through true interaction between the two scales in both space and time: the microscale and the macroscale.

This paper focuses on this challenge, with the objective of reducing calculation costs drastically while, at the same time, trying to improve robustness.

The central issue is the transfer of information from one scale to another. A very efficient strategy for linear periodic media consists in applying the homogenization theory initiated by Sanchez-Palencia $[5,6]$. Further developments and related computational approaches can be found in [7-12]. First, the resolution of the macro problem leads to effective values of the unknowns; then, the micro solution is calculated locally based on the macro solution. The fundamental assumption, besides periodicity, is that the ratio of the characteristic length of the small scale to the characteristic length of the large scale must be small. Boundary zones, in which the material cannot be homogenized, require special treatment. Moreover, this theory is not directly applicable to time-dependent nonlinear problems. Other computational strategies using homogenization techniques based on the Hill-Mandel conditions [13] have also been proposed $[14,15]$ and have similar limitations. Other paradigms for building multiscale computational strategies can be found in $[16,17]$. All these approaches introduce different scales only in space.

Only relatively few works have been devoted to multi-time-scale computational strategies. What are called multi-time-step methods [18-21] and timedecomposed parallel time integrators $[22,23]$ deal with different time discretizations and integration schemes. Local enrichment functions were introduced in [24]. In multiphysics problems, coupling between time grids may be envisaged. This type of problem was solved in [25] through the introduction of "micro/macro projectors" between grids. Parareal [26] or PITA [22] approaches belong in this category. However, none of these strategies involves a true timehomogenization technique. Such a technique seems to have been used only for periodic loading histories [27-35].

Our first attempt to meet our challenge was to devise a new micro/macro computational strategy [17] which involved space homogenization over the whole domain while avoiding the drawbacks of classical homogenization theory. This technique was expanded in [36] to include time as well as space thanks to the LATIN Method, which enables one to work globally over the time-space domain [37]. This is an iterative strategy. Here, it will be described in detail for (visco)plastic materials and optional unilateral contact with or without friction, a case already introduced in [17]. More complex types of material behavior could also be taken into account.

The first characteristic of the method resides in the partitioning of the space- 
time domain. The structure is defined as an assembly of substructures and interfaces. Each component has its own variables and its own equations. The time interval is divided into subintervals, using the discontinuous Galerkin method to handle possible discontinuities. The junction between the macroscale and the microscale takes place only at the interfaces. Each quantity of interest is considered to be the sum of a macro quantity and a micro quantity, where the macro quantities are defined as "mean values" in time and in space, and the associated micro quantities are the complementary parts; this is a choice. An important point is that due to the Saint Venant principle the effects of the micro quantities are localized in space.

The second characteristic of the method is the use of what we call the LATIN method, a nonincremental iterative computational strategy applied over the entire time interval being studied [37]. At each iteration, one must solve a macro problem defined over the entire structure and the entire time interval, along with a family of independent linear problems, each concerning a substructure and its boundary. The latter are "micro" problems in contrast with the "macro" problem which corresponds to the entire structure homogenized in time as well as in space.

The third characteristic of the method concerns the resolution, over the timespace domain, of the numerous micro problems (whose size can be very large) within the cells or substructures. With the LATIN method, a classical approach consists in using radial time-space approximations [37,38], which reduce calculation and storage costs drastically. Here, a new, more efficient and more robust version is introduced. This technique consists in approximating a function defined in the space-time domain by a sum of products of scalar functions of the time variable by functions of the space variable. As the iterative process goes on, the functions of the space variable constructed in this manner constitute a consistent basis which can be reused for successive iterations. Moreover, when dealing with similar substructures such as cells in composites, this basis is common to all the substructures.

After reviewing the bases of the multiscale strategy with space and time homogenization, this paper will focus on suitable approximation techniques for the resolution of the micro and macro problems and particularly on the new radial time-space approximation. Several numerical examples will illustrate the capabilities of the approach presented.

\section{The reference problem}

Under the assumption of small perturbations, let us consider the quasi-static and isothermal evolution of a structure defined in the time-space domain 
$[0, T] \times \Omega$. This structure is subjected to prescribed body forces $\underline{f}_{d}$, to traction forces $\underline{F}_{d}$ over a part $\partial_{2} \Omega$ of the boundary, and to prescribed displacements $\underline{U}_{d}$ over the complementary part $\partial_{1} \Omega$ (see Figure 1 ).

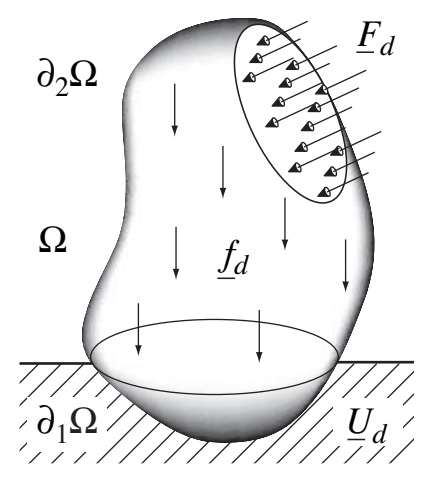

Fig. 1. The reference problem

The state of the structure is defined by the set of the fields $\left(\dot{\varepsilon}_{\mathrm{p}}, \dot{\mathbf{X}}, \boldsymbol{\sigma}, \mathbf{Y}\right)$ (using the dot notation $\dot{\square}$ for the time derivative), in which:

- $\varepsilon_{\mathrm{p}}$ designates the inelastic part of the strain field $\varepsilon$ which corresponds to the displacement field $\underline{U}$, uncoupled into an elastic part $\varepsilon_{\mathrm{e}}$ and an inelastic part $\varepsilon_{\mathrm{p}}=\varepsilon-\varepsilon_{\mathrm{e}} ; \mathbf{X}$ designates the remaining internal variables;

- $\boldsymbol{\sigma}$ designates the Cauchy stress field and $\mathbf{Y}$ the set of variables which are conjugates of $\mathbf{X}$.

All these quantities are defined over the time-space domain $[0, T] \times \Omega$ and assumed to be sufficiently regular. For the sake of simplicity, only the displacement $\underline{U}$ is assumed to have a nonzero initial value, denoted $\underline{U}_{0}$.

Introducing the following notations for the primal fields:

$$
\mathbf{e}_{\mathrm{p}}=\left[\begin{array}{c}
\varepsilon_{\mathrm{p}} \\
-\mathbf{X}
\end{array}\right], \mathbf{e}=\left[\begin{array}{l}
\varepsilon \\
0
\end{array}\right] \text { and } \mathbf{e}_{\mathrm{e}}=\left[\begin{array}{c}
\varepsilon_{\mathrm{e}} \\
\mathbf{X}
\end{array}\right] \text { so that } \mathbf{e}_{\mathrm{p}}=\mathbf{e}-\mathbf{e}_{\mathrm{e}}
$$

and for the dual fields:

$$
\mathbf{f}=\left[\begin{array}{l}
\sigma \\
\mathbf{Y}
\end{array}\right]
$$

the mechanical dissipation rate for the entire structure $\Omega$ is:

$$
\int_{\Omega}\left(\dot{\varepsilon}_{\mathrm{p}}: \boldsymbol{\sigma}-\dot{\mathbf{X}} \cdot \mathbf{Y}\right) d \Omega=\int_{\Omega}\left(\dot{\mathbf{e}}_{\mathrm{p}} \circ \mathbf{f}\right) d \Omega
$$

where denotes the contraction adapted to the tensorial nature of $\mathbf{X}$ and $\mathbf{Y}$, and $\circ$ denotes the corresponding operator. Let us introduce the following 
fundamental "dissipation" bilinear form:

$$
\left\langle\mathbf{s}, \mathbf{s}^{\prime}\right\rangle=\int_{[0, T] \times \Omega}\left(1-\frac{t}{T}\right)\left(\dot{\mathbf{e}}_{\mathrm{p}} \circ \mathbf{f}^{\prime}+\dot{\mathbf{e}}_{\mathrm{p}}^{\prime} \circ \mathbf{f}\right) d \Omega d t
$$

along with $\mathbf{E}$ and $\mathbf{F}$, the spaces of the fields $\dot{\mathbf{e}}_{\mathrm{p}}$ and $\mathbf{f}$ which are compatible with (4). These spaces enable us to define $\mathbf{S}=\mathbf{E} \times \mathbf{F}$, the space in which the state $\mathbf{s}=\left(\dot{\mathbf{e}}_{\mathrm{p}}, \mathbf{f}\right)$ of the structure is being sought.

\subsection{State laws}

Following [37], a normal formulation with internal state variables is used to represent the behavior of the material. If $\rho$ denotes the mass density of the material, from the free energy $\rho \Psi\left(\varepsilon_{\mathrm{e}}, \mathbf{X}\right)$ with the usual uncoupling assumptions, the state law yields:

$$
\begin{gathered}
\boldsymbol{\sigma}=\rho \frac{\partial \psi}{\partial \varepsilon_{\mathrm{e}}}=\mathbf{K} \boldsymbol{\varepsilon}_{\mathrm{e}} \\
\mathbf{Y}=\rho \frac{\partial \psi}{\partial \mathbf{X}}=\boldsymbol{\Lambda} \mathbf{X}
\end{gathered}
$$

where the Hooke's tensor $\mathbf{K}$ and the constant, symmetric and positive definite tensor $\boldsymbol{\Lambda}$ are material characteristics. These equations can be rewritten in the form:

$$
\mathbf{f}=\mathbf{A e}_{\mathrm{e}} \quad \text { with } \quad \mathbf{A}=\left[\begin{array}{cc}
\mathbf{K} & 0 \\
0 & \boldsymbol{\Lambda}
\end{array}\right]
$$

where $\mathbf{A}$ is a constant, symmetric and positive definite operator. Let us note that such an approach is available for most material models [37].

The constitutive equation is given by the positive differential operator $\mathbf{B}$, which is considered to be derived from the dissipation pseudo-potential $\phi^{*}(\boldsymbol{\sigma}, \mathbf{Y})$ :

$$
\dot{\mathrm{e}}_{\mathrm{p}}=\left[\begin{array}{l}
\partial_{\boldsymbol{\sigma}} \phi^{*} \\
\partial_{\mathbf{Y}} \phi^{*}
\end{array}\right]=\mathbf{B}(\mathbf{f}) \quad \text { with } \quad \mathbf{e}_{\mathrm{p} \mid t=0}=0
$$

One should note that for the sake of simplicity we are restricting this presentation to the case of a sufficiently smooth pseudo-potential. Should this not be the case, one would modify (7) by considering $\partial_{\square} \phi^{*}$ to be a subdifferential and replacing the first equality by an inclusion.

For example, if we consider standard viscoplastic behavior with isotropic strain hardening described by the scalar $p$ and kinematic strain hardening described by the second-order tensor $\boldsymbol{\alpha}$, and if the scalar $R$ and the tensor $\boldsymbol{\beta}$ are the 
conjugate variables of $p$ and $\boldsymbol{\alpha}$ respectively, we have:

$$
\begin{aligned}
\rho \psi & =\frac{1}{2} \varepsilon_{\mathrm{e}}: \mathbf{K}: \boldsymbol{\varepsilon}_{\mathrm{e}}+\frac{1}{2} c\|\boldsymbol{\alpha}\|^{2}+\frac{1}{2} \lambda p^{2} \\
\phi^{*} & =\frac{k}{n+1}\left\langle\left\|\boldsymbol{\sigma}_{D}-\boldsymbol{\beta}\right\|+\frac{a}{2 c}\|\boldsymbol{\beta}\|^{2}-\ell(R)-R_{0}\right\rangle_{+}^{n+1}
\end{aligned}
$$

where $\|\boldsymbol{\beta}\|=\sqrt{\boldsymbol{\beta}: \boldsymbol{\beta}}, \boldsymbol{\sigma}_{D}$ is the deviatoric part of Tensor $\boldsymbol{\sigma}$ and $\langle\square\rangle_{+}$extracts the positive part of the argument. Scalars $k, n, c, \lambda, a, R_{0}$ and Function $\ell$ are material characteristics.

\subsection{Compatibility conditions and equilibrium equations}

The compatibility conditions and equilibrium equations are described below and some functional spaces are introduced. We use the notation $\square^{\star}$ to designate the vector space associated with an affine space $\square$.

- The displacement field $\underline{U}$ should match the prescribed displacement $\underline{U}_{d}$ at Boundary $\partial_{1} \Omega$ and the initial condition $\underline{U}_{0}$ at $t=0$ :

$$
\underline{U}_{\mid \partial_{1} \Omega}=\underline{U}_{d} \quad \text { and } \quad \underline{U}_{\mid t=0}=\underline{U}_{0}
$$

The corresponding space of displacement fields $\underline{U}$ is denoted $\mathcal{U}$.

- The stress field $\boldsymbol{\sigma}$ should be symmetric and in equilibrium with the external prescribed forces $\underline{F}_{d}$ at $\partial_{2} \Omega$ and the prescribed body forces $\underline{f}_{d}$ in $\Omega$. The corresponding variational formulation is:

$$
\begin{aligned}
\forall \underline{U}^{\star} \in \mathcal{U}^{\star}, \quad-\int_{[0, T] \times \Omega} \boldsymbol{\sigma}: \varepsilon\left(\underline{\dot{U}}^{\star}\right) d \Omega d t & \\
& \quad+\int_{[0, T] \times \Omega} \underline{f}_{d} \cdot \underline{\dot{U}}^{\star} d \Omega d t+\int_{[0, T] \times \partial_{2} \Omega} \underline{F}_{d} \cdot \underline{\dot{U}}^{\star} d S d t=0
\end{aligned}
$$

The subspace of $\mathbf{F}$ whose elements $\mathbf{f}=[\boldsymbol{\sigma} \mathbf{Y}]^{T}$ verify the previous condition is denoted $\mathcal{F}$. These fields are said to be "statically admissible".

- The strain rate field $\dot{\boldsymbol{\varepsilon}}$ should derive from the symmetric part of the gradient of a displacement field belonging to $\mathrm{Space} \mathcal{U}$. The corresponding variational formulation is:

$$
\forall \mathbf{f}^{\star} \in \mathcal{F}^{\star}, \quad-\int_{[0, T] \times \Omega} \boldsymbol{\sigma}^{\star}: \dot{\varepsilon} d \Omega d t+\int_{[0, T] \times \partial_{1} \Omega} \boldsymbol{\sigma}^{\star} \underline{n} \cdot \underline{\dot{U}}_{d} d S d t=0
$$

The subspace of $\mathbf{E}$ whose elements $\dot{\mathbf{e}}=[\dot{\boldsymbol{\varepsilon}}-\dot{\mathbf{X}}]^{T}$ verify the previous condition is denoted $\mathcal{E}$. These fields are said to be "kinematically admissible". 


\subsection{Formulation of the reference problem}

The reference problem defined over the time-space domain $[0, T] \times \Omega$ can be formulated as follows:

Find $\mathbf{s}_{\mathbf{r e f}}=\left(\dot{\mathbf{e}}_{\mathrm{p}}, \mathbf{f}\right)$ which verifies, with $\mathbf{e}=\mathbf{e}_{\mathrm{e}}+\mathbf{e}_{\mathrm{p}}$,

- the kinematic admissibility $\dot{\mathrm{e}} \in \mathcal{E}$

- the static admissibility $\quad \mathbf{f} \in \mathcal{F}$

- the state law

$\mathbf{f}=\mathrm{Ae}_{\mathrm{e}}$

- the evolution law $\quad \dot{\mathbf{e}}_{\mathrm{p}}=\mathbf{B}(\mathbf{f}) \quad$ with $\quad \mathbf{e}_{\mathrm{p}_{\mid t=0}}=0$

which is equivalent to:

Find $\mathbf{s}_{\mathbf{r e f}}=\left(\dot{\mathbf{e}}_{\mathrm{p}}, \mathbf{f}\right)$ which verifies

$$
\left(\mathbf{A}^{-1} \dot{\mathbf{f}}+\dot{\mathbf{e}}_{\mathrm{p}}\right) \in \mathcal{E}, \quad \mathbf{f} \in \mathcal{F}, \quad \dot{\mathbf{e}}_{\mathrm{p}}=\mathbf{B}(\mathbf{f}) \quad \text { with } \quad \mathbf{e}_{\mathrm{p} \mid t=0}=0
$$

\section{Reformulation of the problem with structure decomposition}

Now, the basic idea consists in describing the structure as an assembly of simple components, i.e. substructures and interfaces, each with its own variables and equations (admissibility, equilibrium and behavior) [37] (see Figure 2).

Each substructure $\Omega_{E}$ of $\Omega$ is defined by the set of variables $\left(\dot{\mathbf{e}}_{\mathrm{p} E}, \mathbf{f}_{E}\right)$ and subjected at its boundary $\partial \Omega_{E}$ to the action of its environment (the neighboring interfaces), described by a displacement distribution $\underline{W}_{E}$ and a force distribution $\underline{F}_{E}$. We will use the subscript $\square_{E}$ to designate the restriction of variables and operators to Subdomain $\Omega_{E}$.

Clearly, $\underline{W}_{E}$ and $\underline{F}_{E}$ viewed from Substructure $\Omega_{E}$ play the role of prescribed boundary conditions. If these boundary conditions are assumed to be known and compatible, the problem in Subdomain $\Omega_{E}$ consists in finding a solution of an equation similar to (13) in which $\underline{W}_{E}$ participates in the definition of kinematic admissibility and $\underline{F}_{E}$ in the definition of static admissibility.

Let $\mathbf{s}_{E}=\left(\dot{\mathbf{e}}_{\mathrm{p} E}, \underline{W}_{E}, \mathbf{f}_{E}, \underline{F}_{E}\right)$ denote the set of the variables describing the state of Substructure $\Omega_{E}$ and its boundary $\partial \Omega_{E}$. The mechanical dissipation rate in Substructure $\Omega_{E}$ is:

$$
\int_{\Omega_{E}}\left(\dot{\mathbf{e}}_{\mathrm{p} E} \circ \mathbf{f}_{E}\right) d \Omega-\int_{\partial \Omega_{E}} \dot{W}_{E} \cdot \underline{F}_{E} d S
$$




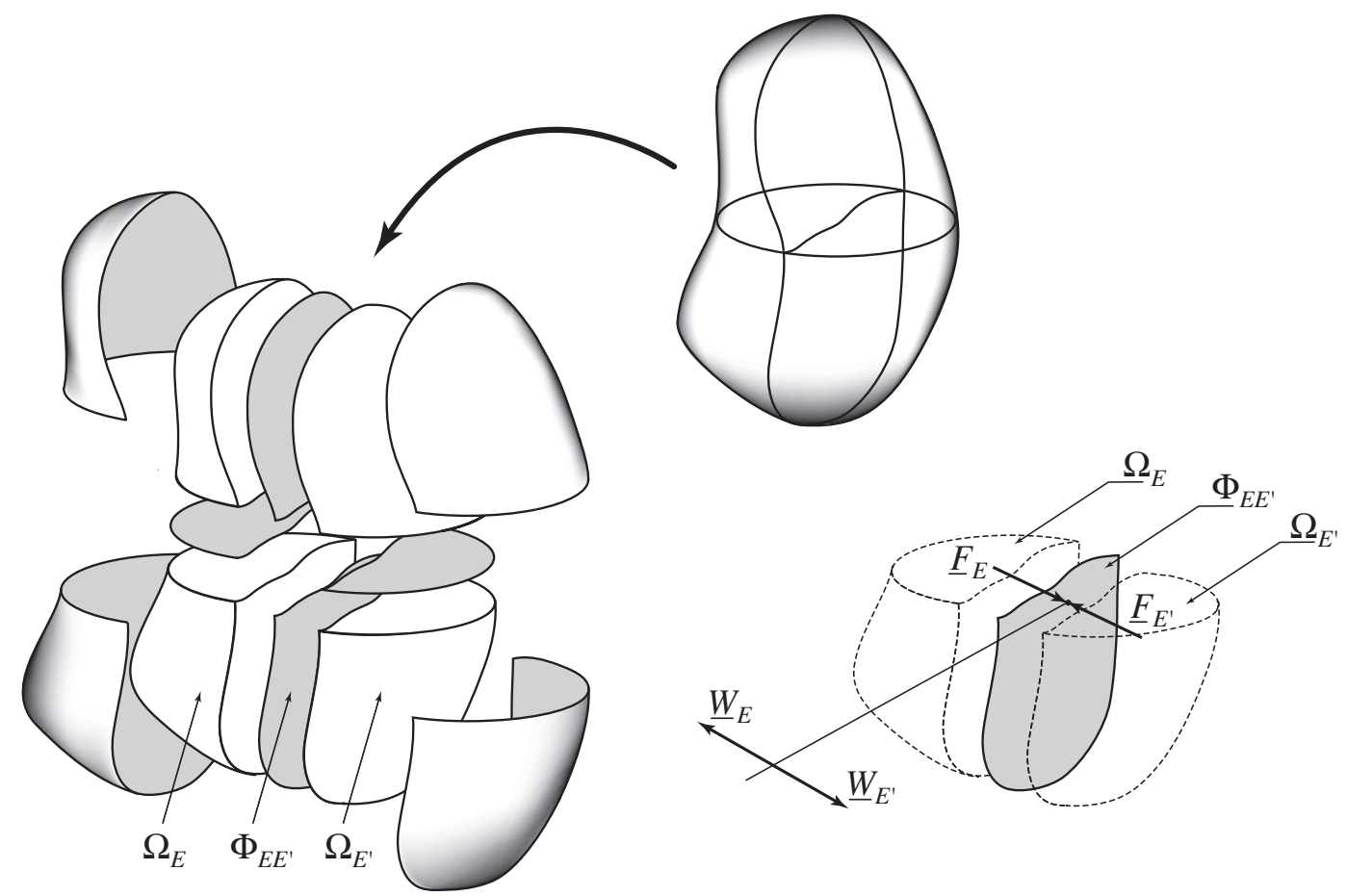

Fig. 2. Decomposition of the structure into substructures and interfaces

and we introduce the following fundamental "dissipation" bilinear form:

$$
\begin{aligned}
\left\langle\mathbf{s}_{E}, \mathbf{s}_{E}^{\prime}\right\rangle_{E}=\int_{[0, T] \times \Omega_{E}}\left(1-\frac{t}{T}\right)\left(\dot{\mathbf{e}}_{\mathrm{p} E} \circ \mathbf{f}_{E}^{\prime}+\dot{\mathbf{e}}_{\mathrm{p} E}^{\prime} \circ \mathbf{f}_{E}\right) d \Omega d t \\
-\int_{[0, T] \times \partial \Omega_{E}}\left(1-\frac{t}{T}\right)\left(\underline{\dot{W}}_{E} \cdot \underline{F}_{E}^{\prime}+\underline{\dot{W}}_{E}^{\prime} \cdot \underline{F}_{E}\right) d S d t
\end{aligned}
$$

along with $\mathbf{E}_{E}, \mathcal{W}_{E}, \mathbf{F}_{E}$ and $\mathcal{F}_{E}$, the spaces of the fields $\dot{\mathrm{e}}_{\mathrm{p} E}, \underline{\underline{W}}_{E}, \mathbf{f}_{E}$ and $\underline{F}_{E}$ which are compatible with (15). These spaces enable us to introduce $\mathbf{S}_{E}=$ $\mathbf{E}_{E} \times \mathcal{W}_{E} \times \mathbf{F}_{E} \times \mathcal{F}_{E}$, the space within which $\mathbf{s}_{E}=\left(\dot{\mathbf{e}}_{\mathrm{p} E}, \underline{W}_{E}, \mathbf{f}_{E}, \underline{F}_{E}\right)$ is being sought.

Partitioning a structure into non-overlapping subdomains is a rather classical idea in mechanics. Another idea consists in considering the interface variables, or at least some of these variables, to be Lagrange multipliers. With this approach, the variables we introduced can be viewed as distributed Lagrange multipliers of both the displacement and force types. One can observe that at each point of an interface one has three displacement-force pairs: one for each substructure on either side of the interface plus one for the interface itself.

One can also note that our description provides a natural framework for dealing with different discretizations in adjacent subdomains (non-matching grids), because the interfaces and subdomains can be meshed independently of one another. 


\subsection{Admissibility conditions for Substructure $\Omega_{E}$}

The following conditions must be verified:

- The displacement field $\underline{U}_{E}$ should match the interface displacement $\underline{W}_{E}$ at Boundary $\partial \Omega_{E}$ and the initial condition $\underline{U}_{E 0}$ at $t=0$ :

$$
\underline{U}_{E \mid \partial \Omega_{E}}=\underline{W}_{E} \quad \text { and } \quad \underline{U}_{E \mid t=0}=\underline{U}_{E 0}
$$

The corresponding space of displacement fields $\left(\underline{U}_{E}, \underline{W}_{E}\right)$ is denoted $\mathcal{U}_{E}$.

- The stress field $\boldsymbol{\sigma}_{E}$ should be symmetric and in equilibrium with the interface forces $\underline{F}_{E}$ on $\partial_{\Omega_{E}}$ and the prescribed body forces $\underline{f}_{d}$ on $\Omega_{E}$. The corresponding variational formulation is:

$$
\begin{aligned}
\forall\left(\underline{U}_{E}^{\star}, \underline{W}_{E}^{\star}\right) \in & \mathcal{U}_{E}^{\star}, \quad-\int_{[0, T] \times \Omega_{E}} \boldsymbol{\sigma}_{E}: \varepsilon\left(\underline{\dot{U}}_{E}^{\star}\right) d \Omega d t \\
& +\int_{[0, T] \times \Omega_{E}} \underline{f}_{d} \cdot \underline{\dot{U}}_{E}^{\star} d \Omega d t+\int_{[0, T] \times \partial \Omega_{E}} \underline{F}_{E} \cdot \underline{\dot{W}}_{E}^{\star} d S d t=0
\end{aligned}
$$

The subspace of $\mathbf{F}_{E} \times \mathcal{F}_{E}$ whose elements $\mathbf{f}_{E}=\left[\boldsymbol{\sigma}_{E} \mathbf{Y}_{E}\right]^{T}$ verify the previous condition is denoted $\mathcal{F}_{E}$.

- The strain rate field $\dot{\varepsilon}_{E}$ should derive from the symmetric part of the gradient of a displacement field belonging to Space $\mathcal{U}_{E}$. The corresponding variational formulation is:

$$
\forall\left(\mathbf{f}_{E}^{\star}, \underline{F}_{E}^{\star}\right) \in \mathcal{F}_{E}^{\star}, \quad-\int_{[0, T] \times \Omega_{E}} \boldsymbol{\sigma}_{E}^{\star}: \dot{\boldsymbol{\varepsilon}}_{E} d \Omega d t+\int_{[0, T] \partial \Omega_{E}} \underline{F}_{E}^{\star} \cdot \underline{\underline{W}}_{E} d S d t=0
$$

The subspace of $\mathbf{E}_{E} \times \mathcal{W}_{E}$ whose elements $\mathbf{e}_{E}=\left[\begin{array}{l}\dot{\varepsilon}_{E}-\dot{\mathbf{X}}_{E}\end{array}\right]^{T}$ verify the previous condition is denoted $\mathcal{E}_{E}$.

- Then, the set of variables $\mathbf{s}_{E}=\left(\dot{\mathbf{e}}_{\mathrm{p} E}, \underline{\dot{W}}_{E}, \mathbf{f}_{E}, \underline{F}_{E}\right)$ should verify:

$$
\left(\mathbf{A}^{-1} \dot{\mathbf{f}}_{E}+\dot{\mathbf{e}}_{\mathrm{p} E}\right) \in \mathcal{E}_{E} \quad \text { and } \quad \mathbf{f}_{E} \in \mathcal{F}_{E}
$$

which defines $\mathbf{A}_{\mathbf{d} E}$, the subspace of $\mathbf{S}_{E}$ whose elements $\mathbf{s}_{E}$ verify the previous conditions (these fields are said to be " $E$-admissible"), but also the evolution law:

$$
\dot{\mathbf{e}}_{\mathrm{p} E}=\mathbf{B}\left(\mathbf{f}_{E}\right) \quad \text { and }\left.\quad \mathbf{e}_{\mathrm{p} E}\right|_{t=0}=0
$$




\subsection{Interface behavior}

The interface concept can be easily extended to the boundary of $\Omega, \partial \Omega$, where either the displacements or the forces are prescribed. It suffices to set:

- for a prescribed displacement at $\Phi_{E 1}=\partial \Omega_{E} \cap \partial_{1} \Omega$ : $\underline{W}_{E}=\underline{U}_{d}$;

- for a prescribed force at $\Phi_{E 2}=\partial \Omega_{E} \cap \partial_{2} \Omega: \quad \underline{F}_{E}=\underline{F}_{d}$.

Let $\Omega_{E}$ denote the set of the neighboring substructures of $\Omega_{E}$ and $\Phi_{E E^{\prime}}$ the interface between $\Omega_{E}$ and $\Omega_{E^{\prime}} \in \Omega_{E}$. This interface is characterized by the restrictions to $\Phi_{E E^{\prime}}$ of both the displacement field $\left(\underline{W}_{E}, \underline{W}_{E^{\prime}}\right)$ and the force field $\left(\underline{F}_{E}, \underline{F}_{E^{\prime}}\right)$, denoted $\left(\underline{W}_{E E^{\prime}}, \underline{W}_{E^{\prime} E}\right)$ and $\left(\underline{F}_{E E^{\prime}}, \underline{F}_{E^{\prime} E}\right)$ respectively. At Interface $\Phi_{E E^{\prime}}$, the action-reaction principle:

$$
\underline{F}_{E E^{\prime}}+\underline{F}_{E^{\prime} E}=\underline{0}
$$

holds, along with a constitutive relation of the form:

$$
\underline{F}_{E E^{\prime} \mid t}=\mathbf{b}_{E E^{\prime}}\left(\left[\underline{\underline{W}}_{E E^{\prime}}-\underline{\dot{W}}_{E^{\prime} E}\right]_{\mid \tau}, \tau \leqslant t\right)
$$

where $\mathbf{b}_{E E^{\prime}}$ is an operator characterizing the behavior of the interface. For instance, one can have:

- for a perfect connection:

$$
\underline{W}_{E E^{\prime}}=\underline{W}_{E^{\prime} E}
$$

which can be interpreted as $\mathbf{b}_{E E^{\prime}}$ being a linear stiffness operator with an infinite norm;

- for unilateral contact without friction:

$$
\left\{\begin{array}{l}
\Pi_{E E^{\prime}} \underline{F}_{E E^{\prime}}=0 \\
\underline{n}_{E E^{\prime}} \cdot\left(\underline{W}_{E E^{\prime}}-\underline{W}_{E^{\prime} E}-\underline{g}_{E E^{\prime}}\right) \geqslant 0, \quad \underline{n}_{E E^{\prime}} \cdot \underline{F}_{E E^{\prime}} \leqslant 0 \\
\left(\underline{n}_{E E^{\prime}} \cdot\left(\underline{W}_{E E^{\prime}}-\underline{W}_{E^{\prime} E}-\underline{g}_{E E^{\prime}}\right)\right)\left(\underline{n}_{E E^{\prime}} \cdot \underline{F}_{E E^{\prime}}\right)=0
\end{array}\right.
$$

where $\underline{n}_{E E^{\prime}}$ is the vector normal to Interface $\Phi_{E E^{\prime}}$ going from Subdomain $\Omega_{E}$ to Subdomain $\Omega_{E^{\prime}}, \Pi_{E E^{\prime}}$ is the corresponding orthogonal projector, and $\underline{g}_{E E^{\prime}}$ is the initial gap between the substructures.

Clearly, in the case of problems with multiple contacts, the philosophy of the method consists in fitting the contact interfaces between the substructures with the material interfaces between the different components of the assembly $[37,39]$. Each individual component can also be partitioned artificially using a perfect connection interface. 


\subsection{Reformulation of the reference problem}

Going back to the reference problem stated at the beginning (13), this problem obviously consists in finding the set $\mathbf{s}=\left(\mathbf{s}_{E}\right)_{\Omega_{E} \subset \Omega}$ in the space $\mathbf{S}=\bigotimes_{\Omega_{E} \subset \Omega} \mathbf{S}_{E}$. Let $\mathbf{E}, \mathcal{W}, \mathbf{F}$ and $\mathcal{F}$ denote the extensions of the previous spaces $\mathbf{E}_{E}, \mathcal{W}_{E}, \mathbf{F}_{E}$ and $\mathcal{F}_{E}$ to the entire problem. For the sake of simplicity, we will use the notation $\left(\dot{\mathbf{e}}_{\mathrm{p}}, \underline{\dot{W}}, \mathbf{f}, \underline{F}\right) \in \mathbf{E} \times \mathcal{W} \times \mathbf{F} \times \mathcal{F}$ to designate a set $\left(\dot{\mathbf{e}}_{\mathrm{p} E}, \underline{W}_{E}, \mathbf{f}_{E}, \underline{F}_{E}\right)_{\Omega_{E} \subset \Omega} \in$ $\mathbf{E}_{E} \times \mathcal{W}_{E} \times \mathbf{F}_{E} \times \mathcal{F}_{E}$.

The decomposed reference problem, defined over the entire time-space domain $[0, T] \times \Omega$, can be formulated as follows:

Find $\mathbf{s}_{\text {ref }}=\left(\mathbf{s}_{E}\right)_{\Omega_{E} \subset \Omega}$ which verifies, $\forall \Omega_{E} \subset \Omega$,

- the $E$-admissibility condition $\mathbf{s}_{E} \in \mathbf{A}_{\mathbf{d} E}$

- the evolution law $\dot{\mathbf{e}}_{\mathrm{p} E}=\mathbf{B}\left(\mathbf{f}_{E}\right) \quad$ with $\left.\quad \mathbf{e}_{\mathrm{p} E}\right|_{t=0}=0$

- the interface behavior $\forall \Omega_{E^{\prime}} \in \Omega_{E}, \underline{F}_{E E^{\prime}}+\underline{F}_{E^{\prime} E}=\underline{0}$ and $\underline{F}_{E E^{\prime} \mid t}=\mathbf{b}_{E E^{\prime}}\left(\left[\underline{\dot{W}}_{E E^{\prime}}-\underline{\dot{W}}_{E^{\prime} E}\right]_{\mid \tau}, \tau \leqslant t\right)$

\section{Multiscale description in the time-space domain $[0, T] \times \Omega$}

\subsection{A two-scale description of the unknowns}

The following idea was initially introduced for multiscale problems in space, then extended to multiscale problems in both time and space in [36]. The approach consists in introducing a two-scale description of the unknowns: these two scales are denoted "macro" and "micro" and concern both space and time. The distinction between the macrolevel and the microlevel is made only at the interfaces.

For the neighboring interfaces of Substructure $\Omega_{E}$, the unknowns $\left(\underline{\dot{W}}_{E}, \underline{F}_{E}\right) \in$ $\mathcal{W}_{E} \times \mathcal{F}_{E}$ are split into:

$$
\underline{\dot{W}}_{E}=\underline{\dot{W}}_{E}^{M}+\underline{\dot{W}}_{E}^{m} \quad \text { and } \quad \underline{F}_{E}=\underline{F}_{E}^{M}+\underline{W}_{E}^{m}
$$

where Superscripts $\square^{M}$ and $\square^{m}$ designate the macro parts and the micro complements of the fields respectively. The spaces corresponding to the macro parts are $\mathcal{W}_{E}^{M}$ and $\mathcal{F}_{E}^{M}$, and the spaces corresponding to the micro parts are $\mathcal{W}_{E}^{m}$ and $\mathcal{F}_{E}^{m}$. The extensions of these spaces to the entire set of interfaces are $\mathcal{W}^{M}, \mathcal{F}^{M}, \mathcal{W}^{m}$ and $\mathcal{F}^{m}$. 
Spaces $\mathcal{W}_{E}^{M}$ and $\mathcal{F}_{E}^{M}$ can be chosen arbitrarily, provided that they are compatible with (15) and that $\mathcal{W}_{E}^{M}$ includes the trace of the rigid body modes on $\partial \Omega_{E}$ (which implies that $\mathcal{F}^{M}$ contains the self-balanced forces). Once these spaces have been chosen, the macro part $\underline{\dot{W}}_{E}^{M}$ of Field $\underline{\dot{W}}_{E} \in \mathcal{W}_{E}$ is defined by:

$$
\forall \underline{F}^{\star} \in \mathcal{F}_{E}^{M}, \quad \int_{[0, T] \times \partial \Omega_{E}}\left(\underline{\dot{W}}_{E}^{M}-\underline{\dot{W}}_{E}\right) \cdot \underline{F}^{\star} d S d t=0
$$

and the macro part $\underline{F}_{E}^{M}$ of Field $\underline{F}_{E} \in \mathcal{F}_{E}$ by:

$$
\forall \underline{W}^{\star} \in \mathcal{W}_{E}^{M}, \quad \int_{[0, T] \times \partial \Omega_{E}}\left(\underline{F}_{E}^{M}-\underline{F}_{E}\right) \cdot \underline{\dot{W}}^{\star} d S d t=0
$$

Consequently, the micro parts are $\underline{\dot{W}}_{E}^{m}=\underline{\dot{W}}_{E}-\underline{\dot{W}}_{E}^{M}$ and $\underline{F}_{E}^{m}=\underline{F}_{E}-\underline{F}_{E}^{M}$, and the scales are uncoupled as follows:

$$
\int_{[0, T] \times \partial \Omega_{E}} \underline{\dot{W}}_{E} \cdot \underline{F}_{E} d S d t=\int_{[0, T] \times \partial \Omega_{E}}\left(\underline{\dot{W}}_{E}^{M} \cdot \underline{F}_{E}^{M}+\underline{\dot{W}}_{E}^{m} \cdot \underline{F}_{E}^{m}\right) d S d t
$$

For space, the macroscale is defined by the characteristic length of the interfaces, which is a priori much larger than the scale of the spatial discretization. For example, the macro parts are defined as affine functions on each interface $\Phi_{E E^{\prime}}$.

For time, the macroscale is associated with a coarse partition $\mathcal{T}_{h}^{M}=\{0=$ $\left.t_{0}^{M}, \ldots, t_{n^{M}}^{M}=T\right\}$ of the time interval $[0, T]$ being studied. Its characteristic time (i.e. the maximum length of a time step) is chosen much larger than the characteristic time of the initial time discretization $\mathcal{T}_{h}=\left\{0=t_{0}, \ldots, t_{n}=T\right\}$. For example, the macro parts are defined as polynomials of degree $p$ in each macro interval $\left.I_{k}^{M}=\right] t_{k}^{M}, t_{k+1}^{M}$ [. Let us note that the choice of functions which are possibly discontinuous implies that one should consider all the equations in the time-discontinuous Galerkin scheme sense [40].

The choices adopted for the definition of the macro quantities are physically sound: these quantities are mean values in time and in space. Fields $\underline{W}_{E}^{M}$ and $\underline{F}_{E}^{M}$ are written at each space-time point $(\underline{M}, t)$ of $\Phi_{E E^{\prime}} \times I_{k}^{M}$ in the form $\sum_{i, j} \alpha_{i j} \underline{e}_{j}^{M}(\underline{M}) f_{i}^{M}(t)$, for which a choice of basis functions $\underline{e}_{j}^{M}$ and $f_{j}^{M}$ is represented in Figures 3 and 4 in the case of a two-dimensional interface.

\subsection{Admissibility of the macro quantities}

An important feature of the multiscale computational strategy presented here is that the transmission conditions at the interfaces are partially verified $a$ 


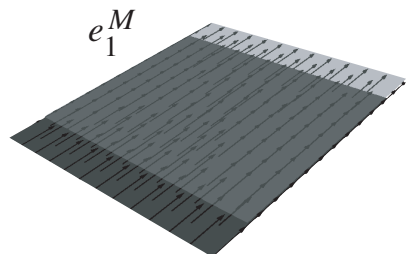

Translation $N_{1}$

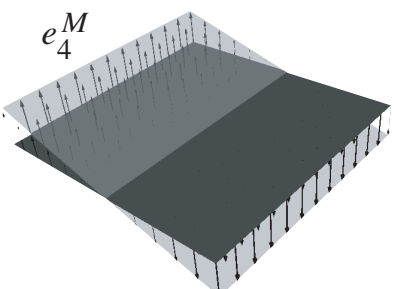

Rotation $N_{1}$

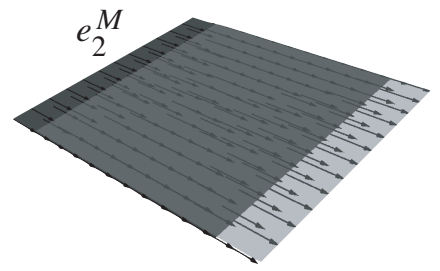

Translation $N_{2}$

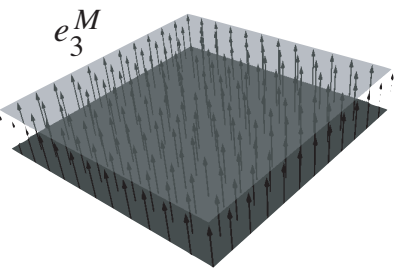

Translation $N_{3}$

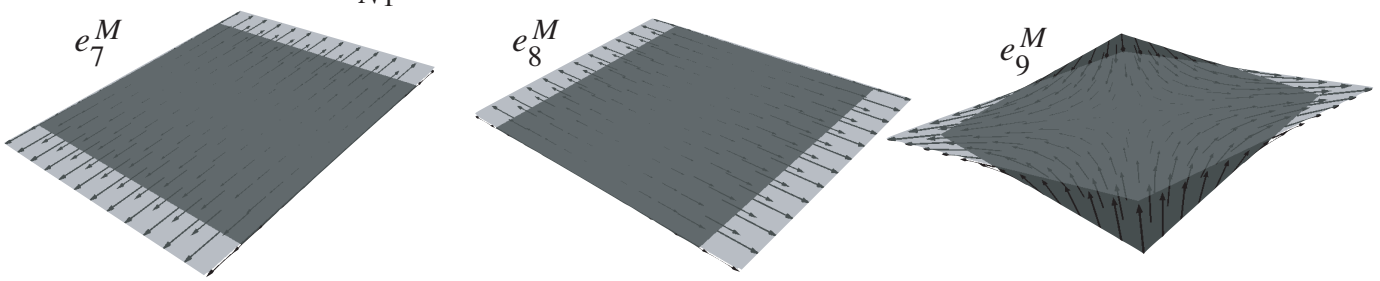

Extension $N_{1}$

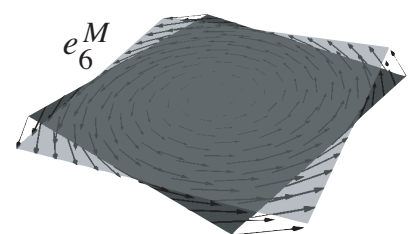

Rotation $N_{3}$

Fig. 3. Space level: affine basis functions $\left\{\underline{e}_{j}^{M}\right\}_{j \in\{1, \ldots, 9\}}$ for an interface $\Phi_{E E^{\prime}}$
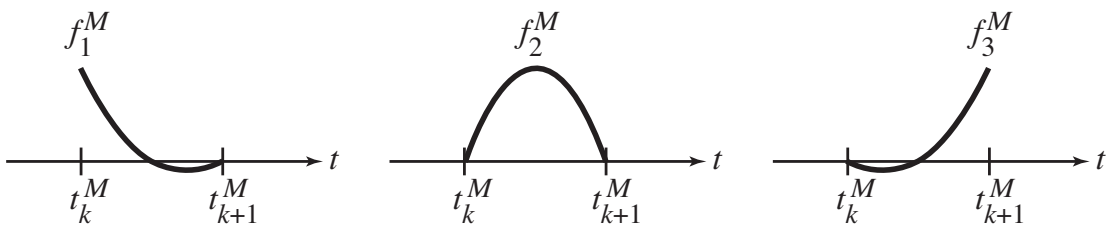

Fig. 4. Time level: quadratic basis functions $(p=2)\left\{f_{i}^{M}\right\}_{i \in\{1, \ldots, 3\}}$ in Interval $I_{k}^{M}$

priori. The set of the macro forces $\underline{F}^{M}=\left(\underline{F}_{E}^{M}\right)_{\Omega_{E} \subset \Omega}$ is required to verify the transmission conditions systematically, including the boundary conditions:

$$
\begin{array}{cl}
\underline{F}_{E E^{\prime}}^{M}+\underline{F}_{E^{\prime} E}^{M}=\underline{0} & \text { on } \Phi_{E E^{\prime}} \\
\underline{F}_{E 2}^{M}+\underline{F}_{d}^{M}=\underline{0} & \text { on } \Phi_{E 2}
\end{array}
$$

The corresponding subspace of $\mathcal{F}^{M}$ is designated by $\mathcal{F}_{\text {ad }}^{M}$. We also introduce $\mathcal{W}_{\text {ad }}^{M}$, the subspace of $\mathcal{W}^{M}$ whose elements are continuous at the interfaces and equal to the prescribed velocity $\underline{\dot{U}}_{d}$ on $\partial_{1} \Omega$. The subspaces of $\mathcal{W}$ and $\mathcal{F}$ whose elements have their macro parts in $\mathcal{W}_{\text {ad }}^{M}$ and $\mathcal{F}_{\text {ad }}^{M}$ are designated by $\mathcal{W}_{\text {ad }}$ and $\mathcal{F}_{\text {ad }}$. 


\section{The multiscale computational strategy}

\subsection{The driving force of the strategy}

The decomposed reference problem, defined over the time-space domain $[0, T] \times$ $\Omega$, can be formulated as follows:

Find $\mathbf{s}_{\text {ref }}=\left(\mathbf{s}_{E}\right)_{\Omega_{E} \subset \Omega}$ which verifies, $\forall \Omega_{E} \subset \Omega$,

(a) the E-admissibility condition $\quad \mathbf{s}_{E} \in \mathbf{A}_{\mathbf{d} E}$

(b) the admissibility of the macro forces $\underline{F} \in \mathcal{F}_{\text {ad }}$

(c) the evolution law $\quad \dot{\mathbf{e}}_{\mathrm{p} E}=\mathbf{B}\left(\mathbf{f}_{E}\right)$ with $\mathbf{e}_{\mathrm{p} E \mid t=0}=0$

(d) the interface behavior $\quad \forall \Omega_{E^{\prime}} \in \Omega_{E}, \underline{F}_{E E^{\prime}}+\underline{F}_{E^{\prime} E}=\underline{0}$ and

$\underline{F}_{E E^{\prime} \mid t}=\mathbf{b}_{E E^{\prime}}\left(\left[\underline{\dot{W}}_{E E^{\prime}}-\underline{\dot{W}}_{E^{\prime} E}\right]_{\mid \tau}, \tau \leqslant t\right)$

The driving force of the strategy we are about to describe is the LATIN method [37], which is a general, mechanics-based computational strategy for the resolution of time-dependent nonlinear problems which works over the entire time-space domain. It has been successfully applied to a variety of problems: quasi-static and dynamic analysis, post-buckling analysis, analysis of highly heterogeneous systems $[39,17,41]$ and multiphysics problems [25].

The first principle of the LATIN method consists in dealing with the difficulties separately by dividing the solutions of the equations into two independent subspaces: the space $\mathbf{A}_{\mathbf{d}}$ of the solutions to the global linear equations (31a) and (31b) (defined on the level of the whole structure) and the space $\boldsymbol{\Gamma}$ of the solutions to the local nonlinear equations (31c) and (31d) (defined on the local level).

The second principle of the method consists in using an iterative scheme to obtain the solution of the problem, which can be interpreted as $\mathbf{s}_{\text {ref }}=\mathbf{A}_{\mathbf{d}} \cap \boldsymbol{\Gamma}$. One iteration consists of two stages, called the "local stage" and the "linear stage". As shown in Figure 5, these stages consist in building fields of $\boldsymbol{\Gamma}$ and $\mathbf{A}_{\mathbf{d}}$ alternatively, an iterative process which, under conditions which will be described later, converges towards the solution $\mathbf{s}_{\text {ref }}$ of the problem. These stages will be analyzed in the following sections.

Figure 6 attempts to give a geometrical interpretation of the method in the space generated by $\left(\dot{\mathbf{e}}_{\mathrm{p}}, \underline{W}\right)$ and $(\mathbf{f}, \underline{F})$, by showing the sets of equations $\mathbf{A}_{\mathbf{d}}$ and $\boldsymbol{\Gamma}$, and the "search directions" $\mathbf{E}^{+}$and $\mathbf{E}^{-}$which are introduced to converge toward the solution. 
$\cdots \longrightarrow \mathbf{s}_{n} \in \mathbf{A}_{\mathbf{d}} \underbrace{\stackrel{\text { local stage }}{\longrightarrow} \hat{\mathbf{s}}_{n+1 / 2} \in \mathbf{\Gamma} \stackrel{\text { linear stage }}{\longrightarrow} \mathbf{s}_{n+1} \in \mathbf{A}_{\mathbf{d}}}_{\text {Iteration } n+1} \longrightarrow \hat{\mathbf{s}}_{n+3 / 2} \in \boldsymbol{\Gamma} \longrightarrow \cdots \longrightarrow \mathbf{s}_{\text {ref }}$

Fig. 5. Local stage and linear stage at Iteration $n+1$

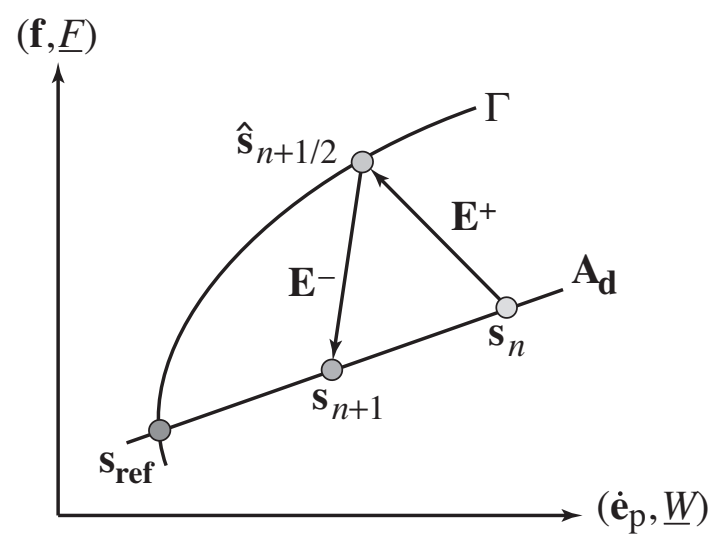

Fig. 6. One iteration of the LATIN method

\subsection{The local stage at Iteration $n+1$}

This stage consists in building $\hat{\mathbf{s}}_{n+1 / 2}=\left(\hat{\mathbf{s}}_{E}\right)_{\Omega_{E} \subset \Omega} \in \boldsymbol{\Gamma}$ knowing $\mathbf{s}_{n}=\left(\mathbf{s}_{E}\right)_{\Omega_{E} \subset \Omega} \in$ $\mathbf{A}_{\mathbf{d}}$ and using an "ascent" search direction $\mathbf{E}^{+}$, followed by $\hat{\mathbf{s}}_{n+1 / 2}-\mathbf{s}_{n}=D \mathbf{s}$ (see Figure 6). This search direction is defined by:

$$
D \mathbf{s}=\left(D \mathbf{s}_{E}\right)_{\Omega_{E} \subset \Omega} \in \mathbf{E}^{+} \Longleftrightarrow \forall \Omega_{E} \subset \Omega,\left\{\begin{array}{r}
D \dot{\mathbf{e}}_{\mathrm{p} E}+\mathbf{H} D \mathbf{f}_{E}=0 \\
D \underline{\dot{W}}_{E}-\mathbf{h} D \underline{F}_{E}=0
\end{array}\right.
$$

where $\mathbf{H}$ and $\mathbf{h}$ are symmetric, positive definite operators which are parameters of the method. This search direction can be redefined using a weak formulation for the substructure part:

$$
\forall \mathbf{f}^{\star} \in \mathbf{F}, \sum_{\Omega_{E} \subset \Omega} \int_{[0, T] \times \Omega_{E}}\left(D \dot{\mathbf{e}}_{\mathrm{p} E}+\mathbf{H} D \mathbf{f}_{E}\right) \circ \mathbf{f}_{E}^{\star} d \Omega d t=0
$$

and for the boundary part:

$$
\forall \underline{F}^{\star} \in \mathcal{F}, \sum_{\Omega_{E} \subset \Omega} \int_{[0, T] \times \partial \Omega_{E}}\left(D \underline{\dot{W}}_{E}-\mathbf{h} D \underline{F}_{E}\right) \cdot \underline{F}_{E}^{\star} d S d t=0
$$

One can easily show that seeking $\hat{\mathbf{s}}_{n+1 / 2}$ common to $\boldsymbol{\Gamma}$ and $\mathbf{E}^{+}$leads to the resolution of a set of problems which are local in the space variable (and, very often, also in the time variable), and, therefore, lend themselves to the highest 
degree of parallelism. This property justifies the term "local" to describe this stage.

\subsection{The linear stage at Iteration $n+1$}

This stage consists in building $\mathbf{s}_{n+1}=\left(\mathbf{s}_{E}\right)_{\Omega_{E} \subset \Omega} \in \mathbf{A}_{\mathbf{d}}$ knowing $\hat{\mathbf{s}}_{n+1 / 2}=$ $\left(\hat{\mathbf{s}}_{E}\right)_{\Omega_{E} \subset \Omega} \in \boldsymbol{\Gamma}$ and using a "descent" search direction $\mathbf{E}^{-}$, followed by $\mathbf{s}_{n+1}-$ $\hat{\mathbf{s}}_{n+1 / 2}=D \mathbf{s}$ (see Figure 6). This search direction is defined by:

$$
D \mathbf{s}=\left(D \mathbf{s}_{E}\right)_{\Omega_{E} \subset \Omega} \in \mathbf{E}^{-} \Longleftrightarrow \forall \Omega_{E} \subset \Omega,\left\{\begin{array}{c}
D \dot{\mathbf{e}}_{\mathrm{p} E}-\mathbf{H} D \mathbf{f}_{E}=0 \\
D \underline{\dot{W}}_{E}+\mathbf{h} D \underline{F}_{E}=0
\end{array}\right.
$$

This search direction can be redefined using a weak formulation for the substructure part:

$$
\forall \mathbf{f}^{\star} \in \mathbf{F}, \sum_{\Omega_{E} \subset \Omega} \int_{[0, T] \times \Omega_{E}}\left(D \dot{\mathbf{e}}_{\mathrm{p} E}-\mathbf{H} D \mathbf{f}_{E}\right) \circ \mathbf{f}_{E}^{\star} d \Omega d t=0
$$

and for the boundary part:

$$
\forall \underline{F}^{\star} \in \mathcal{F}_{\mathbf{a d}}, \quad \sum_{\Omega_{E} \subset \Omega} \int_{[0, T] \times \partial \Omega_{E}}\left(D \underline{\dot{W}}_{E}+\mathbf{h} D \underline{F}_{E}\right) \cdot \underline{F}_{E}^{\star} d S d t=0
$$

with the adjunction of the condition that the test function $\underline{F}^{\star}$ belongs to $\mathcal{F}_{\text {ad }}$ instead of $\mathcal{F}$, which enables one to guarantee the admissibility of the macro forces. The last equation is reformulated with the introduction of a Lagrange multiplier $\underline{\tilde{\tilde{W}}}^{M}\left(\underline{\tilde{\tilde{W}}}^{M}=\left(\underline{\tilde{W}}_{E}^{M}\right)_{\Omega_{E} \subset \Omega} \in \mathcal{W}_{\text {ad }}^{M \star}\right)$ :

$$
\begin{aligned}
\forall \underline{F}^{\star} \in \mathcal{F}, \sum_{\Omega_{E} \subset \Omega}\left\{\int_{[0, T] \times \partial \Omega_{E}}\left(D \underline{\dot{W}}_{E}+\mathbf{h} D \underline{F}_{E}\right) \cdot \underline{F}_{E}^{\star} d S d t\right. \\
\left.\quad-\int_{[0, T] \times \partial \Omega_{E}} \underline{\tilde{W}}_{E}^{M} \cdot \underline{F}_{E}^{\star} d S d t\right\}=0
\end{aligned}
$$

and the admissibility of the macro forces is expressed by:

$$
\begin{aligned}
& \forall \underline{\dot{\tilde{W}}}^{M \star} \in \mathcal{W}_{\text {ad }}^{M \star}, \\
& \quad \sum_{\Omega_{E} \subset \Omega}\left\{\int_{[0, T] \times \partial \Omega_{E}} \underline{\tilde{\tilde{W}}}_{E}^{M \star} \cdot \underline{F}_{E} d S d t-\int_{[0, T] \times \Phi_{E 2}} \underline{\tilde{\tilde{W}}}_{E}^{M \star} \cdot \underline{F}_{d} d S d t\right\}=0
\end{aligned}
$$

The resolution of the linear stage can be divided into two parts: the resolution of a set of micro problems defined over each time-space substructure $[0, T] \times$ 
$\Omega_{E}$, and the resolution of a global macro problem defined over the entire timespace domain $[0, T] \times \Omega$.

\subsubsection{The micro problems defined over each $[0, T] \times \Omega_{E}$ and $[0, T] \times \partial \Omega_{E}$}

Each micro problem associated with $\Omega_{E}$ is a linear evolution equation:

Find $\left(\mathbf{s}_{E}\right)_{\Omega_{E} \subset \Omega}$ which verifies, $\forall \Omega_{E} \subset \Omega$,

- the $E$-admissibility condition $\mathbf{s}_{E} \in \mathbf{A}_{\mathbf{d} E}$

- the search direction

Since (38) is local at Boundary $\partial \Omega_{E}$, the micro problems in the substructures are independent of one another. Since $\mathbf{H}$ and $\mathbf{h}$ are positive definite operators, the micro problem defined over $[0, T] \times \Omega_{E}$ has a unique solution such that:

$$
\mathbf{s}_{E}=\mathbf{s}_{E}^{(1)}+\mathbf{s}_{E}^{(2)}\left(\underline{\tilde{W}}_{E}^{M}\right)
$$

where $\mathbf{s}_{E}^{(1)}$ depends on the additional loading and on the previous approximation of the solution $\hat{\mathbf{s}}_{E}$, and $\mathbf{s}_{E}^{(2)}$ depends linearly on $\underline{\tilde{W}}_{E}^{M}$, which is unknown at this stage. In particular, one has:

$$
\underline{F}_{E}^{M}=\underline{\hat{F}}_{E, d}^{M}+\mathbf{L}_{E} \underline{\tilde{W}}_{E}^{M}
$$

where $\underline{\hat{F}}_{E, d}$ is due to the additional loading and to the previous approximation to the solution, and $\mathbf{L}_{E}$ is a linear operator which can be interpreted as a homogenized behavior operator over the time-space substructure $[0, T] \times \Omega_{E}$. This operator can be calculated by solving a set of micro problems over $[0, T] \times$ $\Omega_{E}$ in which one takes successively for $\underline{\tilde{W}}_{E}^{M}$ the macro basis functions of $\mathcal{W}_{E}^{M}$.

\subsubsection{The macro problem defined over $[0, T] \times \Omega$}

The macro problem defined over the entire time-space domain $[0, T] \times \Omega$ is:

Find $\left(\underline{\tilde{\tilde{W}}}^{M}, \underline{F}^{M}\right)$ which verifies

- the admissibility of the Lagrange multiplier $\underline{\tilde{\tilde{W}}}^{M} \in \mathcal{W}_{\text {ad }}^{M \star}$

- the admissibility of the macro forces $\quad \underline{F} \in \mathcal{F}_{\text {ad }}$

- the homogenized behavior

Introducing (42) into the admissibility condition of the macro forces (39), then 
using the micro-macro uncoupling property (29), one has:

$$
\begin{aligned}
\forall \underline{\tilde{W}}^{M \star} \in \mathcal{W}_{\text {ad }}^{M \star}, \sum_{\Omega_{E} \subset \Omega}\left\{\int_{[0, T] \times \partial \Omega_{E}} \underline{\tilde{W}}_{E}^{M \star} \cdot\left(\underline{\hat{F}}_{E, d}^{M}+\mathbf{L}_{E} \underline{\tilde{W}}_{E}^{M}\right) d S d t\right. \\
\left.\quad-\int_{[0, T] \times \Phi_{E 2}} \underline{\tilde{W}}_{E}^{M \star} \cdot \underline{F}_{d} d S d t\right\}=0
\end{aligned}
$$

which corresponds to the resolution of a homogenized problem over the whole structure. If the number of macro time-space substructures is large, an approximation technique based on the introduction of a third scale can be used $[36]$.

\subsubsection{Resolution of the linear stage}

The resolution of the linear stage proceeds as follows: first, one solves a series of micro problems, each defined over $[0, T] \times \Omega_{E}$, in which one takes into account only the data $\hat{\mathbf{s}}_{E}$ of the previous stage. This leads to $\mathbf{s}_{E}^{(1)}$. Then, one solves the macro problem defined over $[0, T] \times \Omega$, leading to $\underline{\tilde{W}}^{M}$. Finally, in order to obtain $\mathbf{s}_{E}^{(2)}$, one solves a second series of micro problems with the Lagrange multiplier as the only data.

Since the macro mesh is defined in time and in space, the micro problems are independent not only from one substructure to another, but also from one macro time interval to another. One should note that the macro quantities are defined at the interfaces only. By treating the medium as a Cosserat material, one can define macro stresses, macro strains... inside a substructure $\Omega_{E}$. Each cell is assumed to be homogeneous on the macroscale. Thus, macro quantities and conjugate quantities could be derived from the generalized forces and displacements at the interfaces, which would lead to a nonconventional Cosserat-like material.

\subsection{Choice of the parameters $(\mathbf{H}, \mathbf{h})$ and convergence of the algorithm}

Following the proof given in [37], one can prove that the quantity $\frac{1}{2}\left(\mathbf{s}_{n+1}+\right.$ $\mathbf{s}_{n}$ ) converges towards $\mathbf{s}_{\mathbf{r e f}}$, the solution of Problem (31). The choice of the parameters $(\mathbf{H}, \mathbf{h})$ influences only the convergence of the algorithm, but does not affect the solution.

To ensure the convergence of $\mathbf{s}_{n}$ and, more generally, to ensure convergence for many types of material behavior, a relaxation technique may be needed. Renaming $\overline{\mathbf{s}}_{n+1}$ the quantity previously denoted $\mathbf{s}_{n+1}$, we redefine $\mathbf{s}_{n+1}$, the 
approximation generated by the linear stage $n+1$, as:

$$
\mathbf{s}_{n+1}=\mu \overline{\mathbf{s}}_{n+1}+(1-\mu) \mathbf{s}_{n}
$$

where $\mu$ is a relaxation parameter usually equal to 0.8 .

In the case of linear behavior, one can choose, for example, $\mathbf{H}=\mathbf{B}$ and $\mathbf{h}=\frac{L}{E T} \mathbf{I}$, where $E$ is the Young's modulus of the material, $L_{E}$ a characteristic length of the interfaces, $T$ the duration of the phenomenon being studied and $\mathbf{I}$ the identity operator. Other possible choices, especially in the nonlinear case, are discussed in [37].

Since the reference solution $\mathbf{S}_{\text {ref }}$ is the intersection of $\boldsymbol{\Gamma}$ and $\mathbf{A}_{\mathbf{d}}$, the distance between $\hat{\mathbf{s}}_{n+1 / 2}$ and $\mathbf{s}_{n}$ is a good error indicator to verify the convergence of the algorithm [42]. The simplest measure of this distance is:

$$
\eta=\frac{\left\|\hat{\mathbf{s}}_{n+1 / 2}-\mathbf{s}_{n}\right\|}{\frac{1}{2}\left\|\hat{\mathbf{s}}_{n+1 / 2}+\mathbf{s}_{n}\right\|}
$$

with:

$$
\|\mathbf{s}\|^{2}=\frac{1}{2} \sum_{\Omega_{E} \subset \Omega} \int_{[0, T] \times \Omega_{E}}\left(1-\frac{1}{T}\right)\left(\dot{\mathbf{e}}_{\mathrm{p} E} \circ \mathbf{H}^{-1} \dot{\mathbf{e}}_{\mathrm{p} E}+\mathbf{f}_{E} \circ \mathbf{H} \mathbf{f}_{E}\right) d \Omega d t
$$

\subsection{First example}

Let us consider the 3D problem of a composite structure containing cracks (see Figure $7(\mathrm{a}))$. The structure is fixed at the bottom and subjected to forces $\underline{F}_{1}$, $\underline{F}_{3}$ and $\underline{F}_{3}$ (see Figure $7(\mathrm{~b})$ ). The overall dimensions are $120 \times 120 \times 20 \mathrm{~mm}$, and the time interval being studied is $T=10 \mathrm{~s}$. The cracks are described using unilateral contact with Coulomb friction characterized by Parameter $f=0.3$.

The structure consists of two types of cells: Type-I cells are homogeneous, made of Type- 1 material; Type-II cells consist of a matrix of Type- 1 material with inclusions of Type- 2 material. Type- 1 and Type- 2 materials are viscoelastic and their properties are given in Table 1. The corresponding constitutive relations are $\dot{\boldsymbol{\varepsilon}}_{p}=\mathbf{B}_{i} \boldsymbol{\sigma}=\frac{1}{\eta_{i}} \mathbf{K}_{i}^{-1} \boldsymbol{\sigma}$.

The problem was divided into 351 substructures and 1,296 interfaces as shown on Figure 8, each substructure corresponding to one cell. On the microlevel, Type-I and Type-II substructures and interfaces were meshed with 847, 717 and 144 degrees of freedom (DOFs) respectively. The distinction between the macroscale and the microscale was made only at the interfaces and the macro part consisted of a single linear element with only 9 DOFs per interface (see 


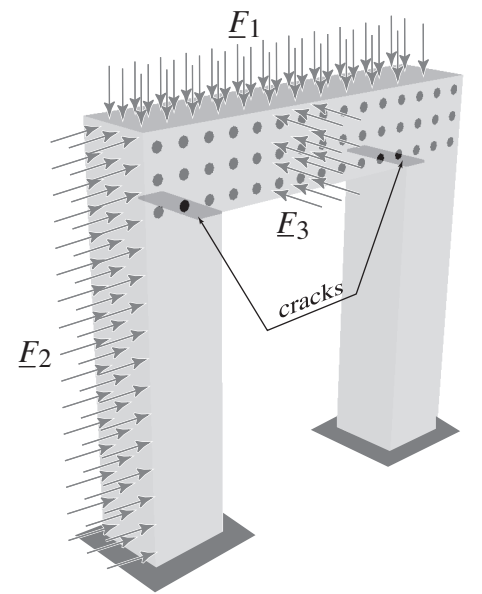

(a) Geometry and loading

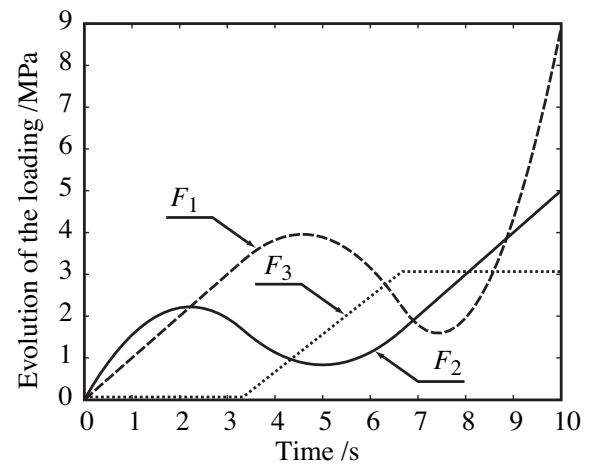

(b) Loads $\underline{F}_{1}, \underline{F}_{3}$ and $\underline{F}_{3}$

Fig. 7. Description of the problem

Table 1

\begin{tabular}{lll}
\hline Material & Type- 1 & Type- 2 \\
\hline Young's modulus & $E_{1}=50 \mathrm{GPa}$ & $E_{2}=250 \mathrm{GPa}$ \\
Poisson's ratio & $\nu_{1}=0.3$ & $\nu_{2}=0.2$ \\
Viscosity parameter & $\eta_{1}=10 \mathrm{~s}$ & $\eta_{2}=1000 \mathrm{~s}$ \\
\hline
\end{tabular}

Material properties

Figure 3). With respect to time, the microlevel was associated with a refined discretization into 60 intervals using a zero-order discontinuous Galerkin scheme, and the macrolevel was associated with a coarse discretization into 3 macro intervals using a second-order discontinuous Galerkin scheme.

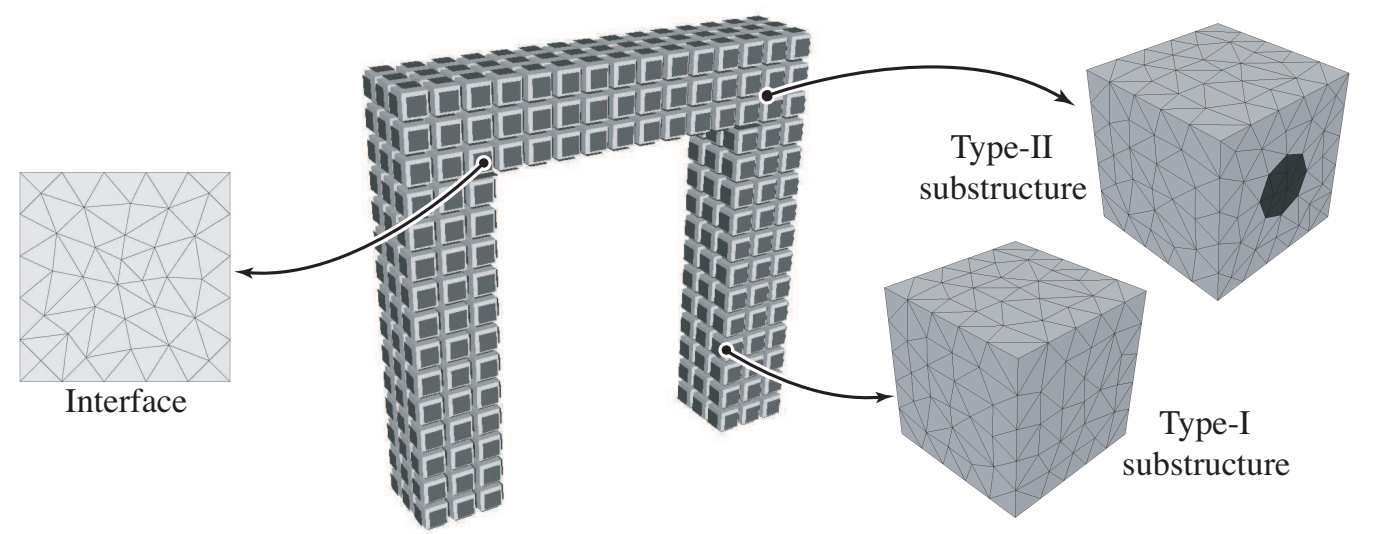

Fig. 8. Decomposition and microscale discretizations in space

Since the constitutive relation is linear, the search direction chosen for the substructures was $\mathbf{H}=\mathbf{B}$. The characteristic length of the interfaces being $L_{E}=4 \mathrm{~mm}$, we chose for all the interfaces the search direction $\mathbf{h}=h \mathbf{I}$, where $h=\frac{L_{E}}{E_{1} \nu_{1}}$ is a constant scalar. 
Figure 9 shows the evolution of the error indicator $\eta$ throughout the iterations. One can observe that the algorithm converges rapidly toward an accurate solution (1\% error after 12 iterations). Figure 10 shows the approximate Von Mises stress field over the structure (with a zoom near one of the cracks) at the final time $T=10 \mathrm{~s}$ for Iterations 1,5 and 20 and after convergence (the reference solution). The evolution over time of the displacement field $\underline{W}$ at Point $P_{2}$ is also represented. One can observe that thanks to the resolution of a macro problem the method leads, even on the first iteration, to a rather good approximation of the solution of the problem over both the space and time domains. After a few iterations, the solution becomes even more accurate and the stress and displacement discrepancies tend to zero. After 20 iterations, the difference between the approximate solution and the reference solution is no longer visible.

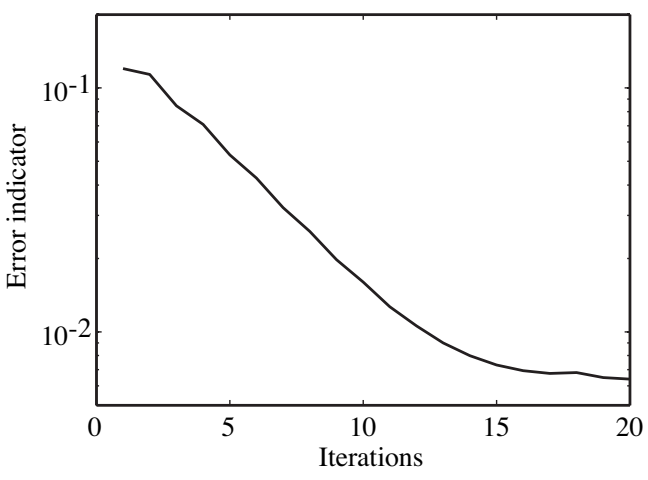

Fig. 9. Convergence of the method

An example of the micro/macro description of the solution is given in Figure 11. Figures 11(a) and 11(b) show the evolutions of Force $\underline{F}$ and its macro part $\underline{F}^{M}$ respectively at time $t=2 / 3 T$ over a horizontal line $L_{1}$ in the heterogeneous part of the structure, and as functions of time at a point $P_{1}$ of the previous line. Figures 11(a) and 11(b) show the same evolutions for Displacement $\underline{W}$ and its macro part $\underline{W}^{M}$.

One can observe that the macro part of the quantities being considered constitutes a good average approximation of the solution, obtained with only a very small number of basis functions (27 DOFs per interface and per macro interval). The choice of such a basis leads to the resolution at each iteration of a macro problem with a strong mechanical meaning and with only a few DOFs (in this example, 35,000 DOFs compared to 270,000 DOFs for the assembled reference problem). 

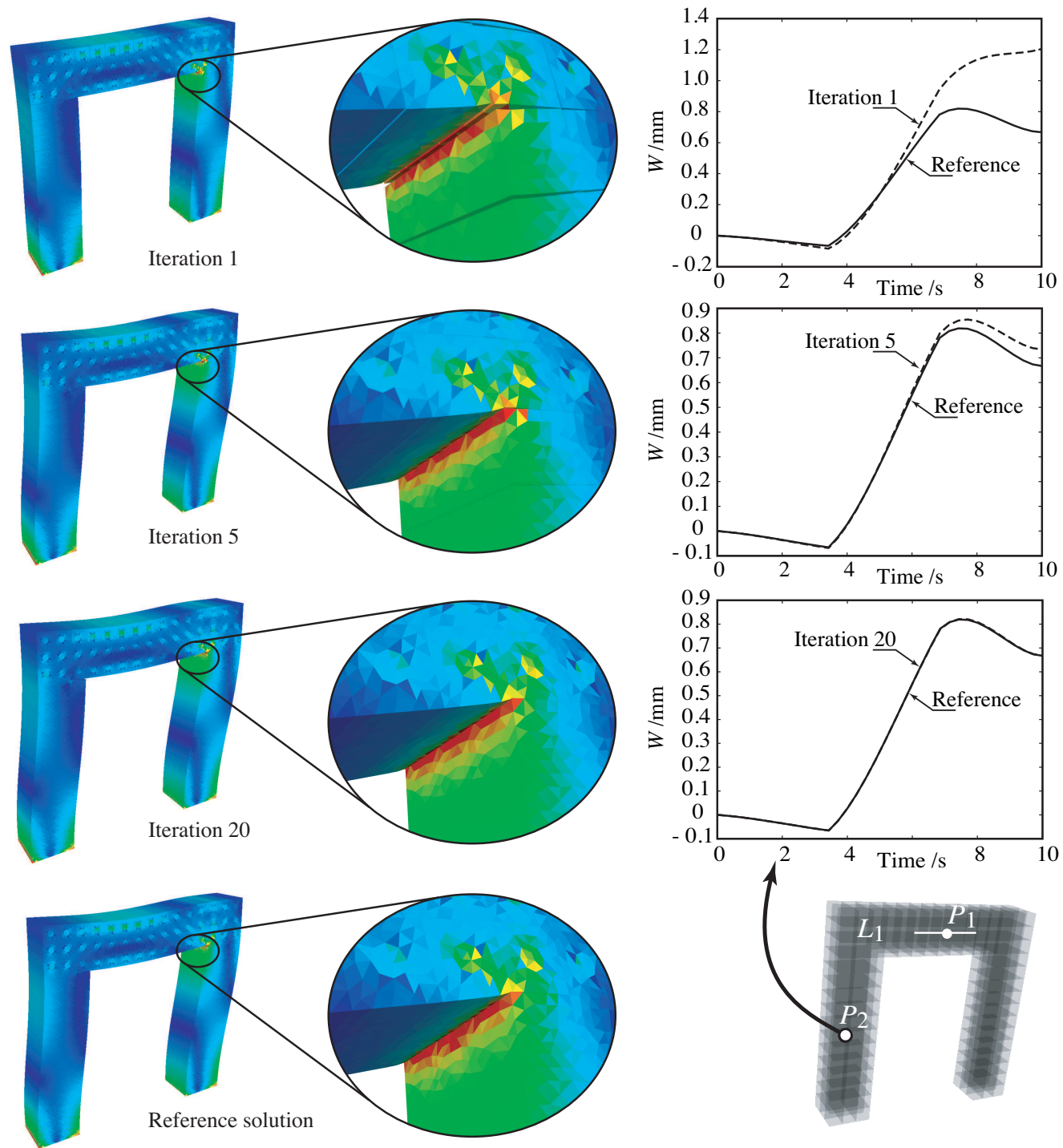

Fig. 10. Approximate solutions throughout the iterations

\section{The radial time-space approximation}

The global structure is decomposed into several substructures. Throughout the iterative process, one has to solve for each of these substructures a set of micro problems which represent the equations defined over the corresponding timespace domains. The cost of solving these problems with standard methods can be prohibitive, which led us to the development of what we call the "radial time-space approximation".

The radial time-space approximation was introduced by Ladevèze in 1985 ([43], see also [34,37]) and is commonly used in the LATIN method. This is the third principle of the LATIN method and it is indeed what makes it so efficient. It was shown in previous works that under the small-displacement 


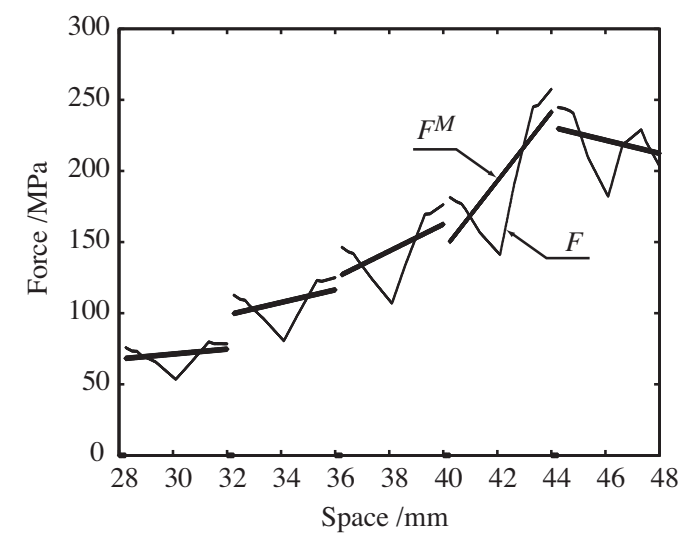

(a) $\underline{F}$ and $\underline{F}^{M}$ at $t=2 / 3 T$ over Line $L_{1}$

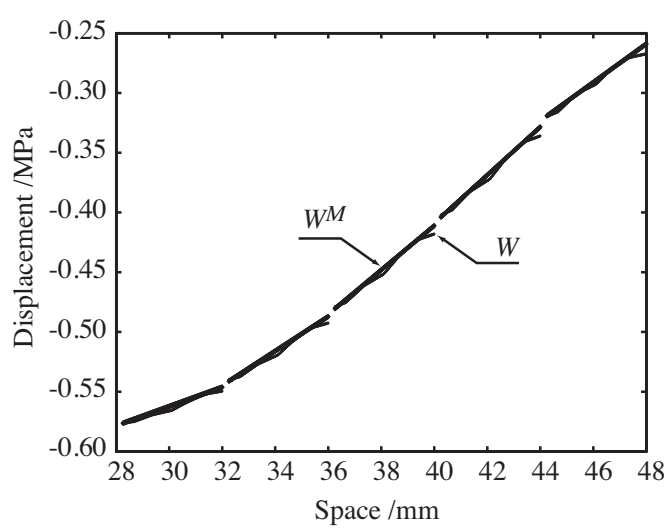

(c) $\underline{W}$ and $\underline{W}^{M} \quad t=2 / 3 T$ over Line $L_{1}$

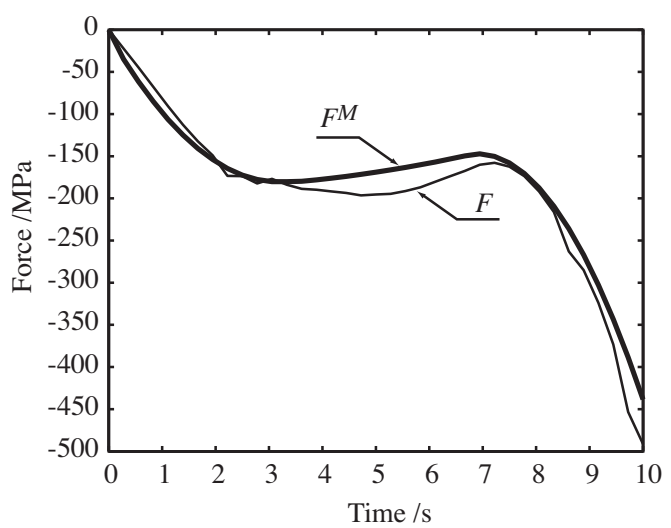

(b) $\underline{F}$ and $\underline{F}^{M}$ at Point $P_{1}$ as functions of time

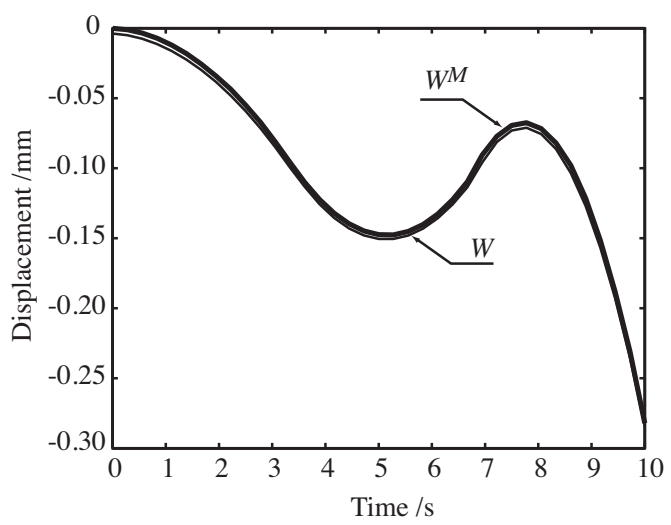

(d) $\underline{W}$ and $\underline{W}^{M}$ at Point $P_{1}$ as functions of time

Fig. 11. Micro/macro description of the solution

assumption this approach reduces the computational cost drastically. The basic idea consists in approximating a function $f$ defined over the time-space domain $[0, T] \times \Omega$ by a finite sum of products of time functions $\lambda_{i}$ by space functions $\Lambda_{i}$ :

$$
\forall(t, M) \in[0, T] \times \Omega, \quad f(t, M): \sum_{i} \lambda_{i}(t) \Lambda_{i}(\underline{M})
$$

where the products $\lambda_{i}(t) \Lambda_{i}(\underline{M})$ are called "radial time-space functions". It is important to note that this is not a spectral decomposition because neither the $\lambda_{i}$ nor the $\Lambda_{i}$ are known a priori.

The starting point of the radial time-space approximation is the radial loading approximation, defined by a single product, which is very well-known and commonly used in (visco-)plasticity. This type of approximation could also be seen by replacing the time variable by space variables or stochastic variables. Such developments have been proposed in [44] for radial hyperreduction, in $[45,46]$ for the resolution of fundamental physics problems and in [47] for the 
resolution of stochastic problems. In a certain way, one can say that such approximations belong to the "Proper Orthogonal Decomposition" class of problems [48].

\subsection{General properties}

Let $f$ be a known scalar function defined over the time-space domain $[0, T] \times \Omega$, and let us study the best $m^{\text {th }}$-order time-space approximation of Function $f$ :

$$
f_{p}(t, \underline{M})=\sum_{i=1}^{p} \lambda_{i}(t) \Lambda_{i}(\underline{M})
$$

The following scalar products are introduced:

$$
\langle f, g\rangle_{[0, T] \times \Omega}=\int_{[0, T] \times \Omega} f g d \Omega d t, \quad\langle f, g\rangle_{[0, T]}=\int_{[0, T]} f g d t, \quad\langle f, g\rangle_{\Omega}=\int_{\Omega} f g d \Omega
$$

It was shown in [37] that the best approximation with respect to the $\|\cdot\|_{[0, T] \times \Omega^{-}}$ norm is the result of an eigenvalue problem whose eigenfunctions are the time functions $\lambda_{i}$. This problem can be rewritten as the stationarity of the Rayleigh quotient:

$$
R(\lambda)=\frac{\left\|\langle f, \lambda\rangle_{[0, T]}\right\|_{\Omega}^{2}}{\|\lambda\|_{[0, T]}^{2}}
$$

It was also proved in [37] that if $[0, T] \times \Omega$ is the space such that $f$ and $\dot{f}$ belong to $L^{2}\left([0, T], L^{2}(\Omega)\right)$, the eigenvalue problem has a countable sequence of eigensolutions $\left(\alpha_{i}^{-1}, \lambda_{i}\right)$ where the eigenvalues $\alpha_{i}^{-1}$ are positive and the eigenfunctions $\lambda_{i}$ are orthogonal.

The time functions $\lambda_{i}$ having been determined, the corresponding space functions $\Lambda_{i}$ are:

$$
\Lambda_{i}=\frac{\left\langle f, \lambda_{i}\right\rangle_{[0, T]}}{\left\|\lambda_{i}\right\|_{[0, T]}^{2}}
$$

The following convergence property is verified:

$$
\left\|f-f_{p}\right\|_{[0, T] \times \Omega} \underset{p \rightarrow+\infty}{\longrightarrow} 0
$$

and a simple measure of the relative error is:

$$
\eta_{p}=\frac{\left\|f-f_{p}\right\|_{[0, T] \times \Omega}}{\left\|\frac{1}{2}\left(f+f_{p}\right)\right\|_{[0, T] \times \Omega}}
$$




\subsection{Illustration}

In order to illustrate the relevance of the previous time-space approximation, let us take as an example the case of a randomly-obtained irregular function $f$ defined over a time-space interval $[0, T] \times[0, L]$. Figure 12 shows Function $f$ along with its first-, second- and third-order approximations. The relative error achieved with only 3 radial functions was less than 1\%, which gives an idea of the remarkable accuracy of the proposed time-space approximation.

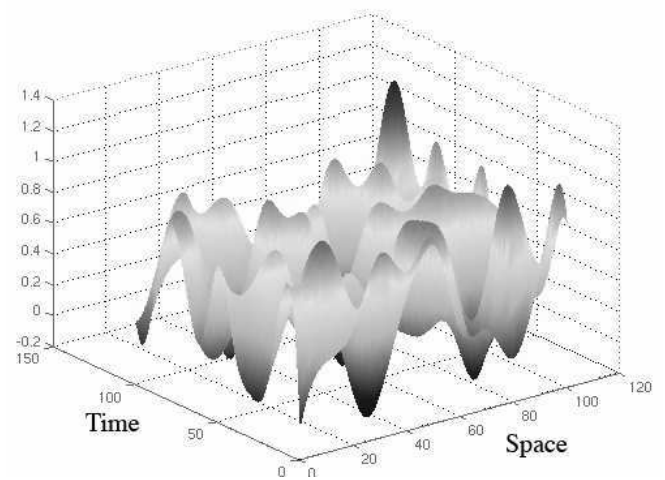

(a) Irregular time-space function $f$

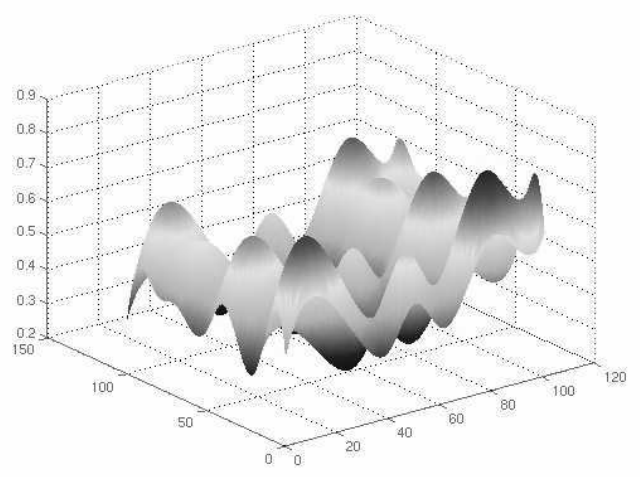

(b) First-order approximation $f_{1}$ : error $\eta_{1}=3.9 \%$

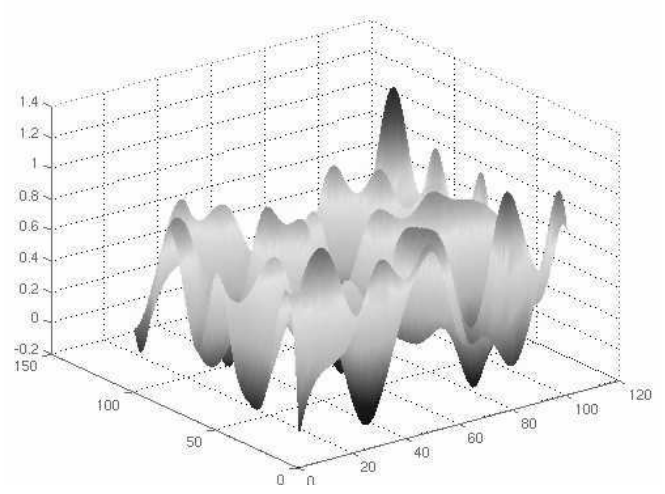

(d) Third-order approximation $f_{3}$ : error $\eta_{3}=0.6 \%$

(c) Second-order approximation $f_{2}$ : error $\eta_{2}=1.5 \%$

Fig. 12. Time-space approximations of an irregular function $f$

\subsection{Practical implementation}

Working with the radial time-space description alone constitutes a very convenient framework in which the storage requirement is drastically reduced. Here, we are following [49] to show the potential of this framework.

Let us divide the time interval $[0, T]$ being studied into $m$ subintervals $\left\{I_{i}\right\}_{i=1, \ldots, m}$ 
of lengths $\left\{\Delta t_{i}\right\}_{i=1, \ldots, m}$, as shown in Figure 13. The centers $\left\{t_{i}\right\}_{i=1, \ldots, m}$ of these subintervals are called "reference times" and one has $I_{i}=\left[t_{i}-\Delta t_{i} / 2, t_{i}+\right.$ $\left.\Delta t_{i} / 2\right]$.

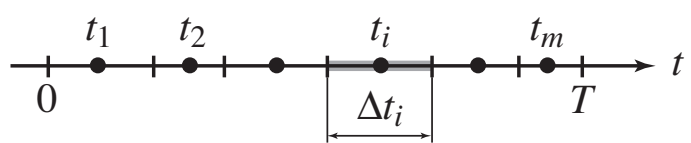

Fig. 13. The reference times in $[0, T]$

For space, let us also introduce $m^{\prime}$ points $\left\{\underline{M}_{j}\right\}_{j=1, \ldots, m^{\prime}}$ and partition $\Omega$ into $\left\{\Omega_{j}\right\}_{j=1, \ldots, m^{\prime}}$, as shown in Figure 14. These points are called "reference points" and the measures of the subdomains are denoted $\left\{\omega_{j}\right\}_{i=j, \ldots, m^{\prime}}$. In practice, there should be about a few dozen reference points.

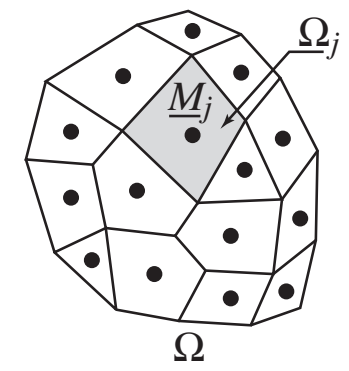

Fig. 14. The reference points in $\Omega$

The choice of these reference times and points is unrelated to the classical discretizations of the time interval $[0, T]$ and domain $\Omega$. Refined time and space discretizations should still be used for the calculation of the various quantities. What we are doing here describes a field $f$ over the time-space domain $[0, T] \times \Omega$ through:

$$
\hat{a}_{i}^{j}(t)=\left\{\begin{array}{ll}
f\left(t, \underline{M}_{j}\right) & \text { if } t \in I_{i} \\
0 & \text { otherwise }
\end{array} \quad \text { and } \quad \hat{b}_{i}^{j}(\underline{M})= \begin{cases}f\left(t_{i}, \underline{M}\right) & \text { if } \underline{M} \in \Omega_{j} \\
0 & \text { otherwise }\end{cases}\right.
$$

for $i=1, \ldots, m$ and $j=1, \ldots, m^{\prime}$.

The sets $\left\{\left(\hat{a}_{i}^{j}, \hat{b}_{i}^{j}\right)\right\}_{i=1, \ldots, m}^{j=1, \ldots, m^{\prime}}$ are the generalized components of $f$. One should note that these quantities verify the following compatibility conditions: for $i=1, \ldots, m$ and $j=1, \ldots, m^{\prime}$,

$$
\hat{a}_{i}^{j}\left(t_{i}\right)=\hat{b}_{i}^{j}\left(\underline{M}_{j}\right)
$$

The main question is then how to build or rebuild a field from its components. We choose to defined Function $f$ from its components by only one product 
per time-space subdomain $I_{i} \times \Omega_{j}$ :

$$
f(t, \underline{M}): a_{i}^{j}(t) b_{i}^{j}(\underline{M}) \quad \forall(t, \underline{M}) \in I_{i} \times \Omega_{j}
$$

where the sets $\left\{\left(a_{i}^{j}, b_{i}^{j}\right)\right\}_{i=1, \ldots, m}^{j=1, \ldots, m^{\prime}}$ should be defined from the sets $\left\{\left(\hat{a}_{i}^{j}, \hat{b}_{i}^{j}\right)\right\}_{i=1, \ldots, m}^{j=1, \ldots, m^{\prime}}$. However, here, we let the time domain play a special role because there are many more spatial degrees of freedom than time degrees of freedom. Then, Function $f$ is defined by:

$$
f(t, \underline{M}): a_{i}(t) b_{i}(\underline{M}) \quad \forall(t, \underline{M}) \in I_{i} \times \Omega
$$

Let us introduce the following scalar products:

$$
\langle f, g\rangle_{I_{i}}=\int_{I_{i}} f g d t \quad \text { and } \quad\langle f, g\rangle_{\Omega_{j}}=\int_{\Omega_{j}} f g d \Omega
$$

In order to get the sets $\left\{\left(a_{i}, b_{i}\right)\right\}_{i=1, \ldots, m}$, we minimize:

$$
J\left(a_{i}, b_{i}\right)=\sum_{j=1}^{m^{\prime}}\left[\omega_{j}\left\|\hat{a}_{i}^{j}(t)-a_{i}(t) b_{i}\left(\underline{M}_{j}\right)\right\|_{I_{i}}^{2}+\Delta t_{i}\left\|\hat{b}_{i}^{j}(\underline{M})-a_{i}\left(t_{i}\right) b_{i}(\underline{M})\right\|_{\Omega_{j}}^{2}\right]
$$

which leads to:

$$
a_{i}(t)=\frac{\sum_{j=1}^{m^{\prime}} \omega_{j} \hat{a}_{i}^{j}(t) b_{i}\left(\underline{M}_{j}\right)}{\sum_{j=1}^{m^{\prime}} \omega_{j} b_{i}^{2}\left(\underline{M}_{j}\right)} \quad \text { and } \quad b_{i}(\underline{M})=\frac{\sum_{j=1}^{m^{\prime}} \hat{b}_{i}^{j}(\underline{M})}{m^{\prime} a_{i}\left(t_{i}\right)}
$$

Consequently, $\forall(t, \underline{M}) \in I_{i} \times \Omega_{j}$, we obtain:

$$
f(t, \underline{M}): a_{i}(t) b_{i}(\underline{M})=\frac{\sum_{k=1}^{m^{\prime}} \omega_{k} \hat{a}_{i}^{k}(t) \hat{b}_{i}^{k}\left(\underline{M}_{k}\right)}{\sum_{k=1}^{m^{\prime}} \omega_{k} \hat{b}_{i}^{k}\left(\underline{M_{k}}\right) \hat{b}_{i}^{k}\left(\underline{M}_{k}\right)} \hat{b}_{i}^{j}(\underline{M})
$$

Then, using the compatibility conditions (56), we get:

$$
f(t, \underline{M}): a_{i}(t) b_{i}(\underline{M})=\frac{\sum_{k=1}^{m^{\prime}} \omega_{k} \hat{a}_{i}^{k}(t) \hat{a}_{i}^{k}\left(t_{i}\right)}{\sum_{k=1}^{m^{\prime}} \omega_{k} \hat{a}_{i}^{k}\left(t_{i}\right) \hat{a}_{i}^{k}\left(t_{i}\right)} \hat{b}_{i}^{j}(\underline{M})
$$

\subsection{Reformulation of the linear stage at Iteration $n+1$}

\subsubsection{Rewriting of a micro problem over $[0, T] \times \Omega_{E}$}

We choose to rewrite the linear stage at Iteration $n+1$ as an incremental correction $\Delta \mathbf{s}$ to the previous approximation $\mathbf{s}_{n}$, so that the new approximation to the solution is $\mathbf{s}_{n+1}=\mathbf{s}_{n}+\Delta \mathbf{s}$. If the initial solution $\mathbf{s}_{0}$ (for example, 
the solution of a linear elastic calculation) belongs to $\mathbf{A}_{\mathbf{d}}$, then all the corrections are sought in $\mathbf{A}_{\mathbf{d}}^{\star}$, the space which corresponds to $\mathbf{A}_{\mathbf{d}}$ with homogeneous conditions.

For each $[0, T] \times \Omega_{E}$, the search direction (35) can be interpreted as a linear constitutive relation. Thus, an equivalent formulation consists in minimizing the global constitutive relation error in $\mathbf{A}_{\mathbf{d} E}^{\star}$, which is defined over the timespace substructure $[0, T] \times \Omega_{E}$. Then, rewriting

$$
D \mathbf{s}_{E}=\mathbf{s}_{E, n+1}-\hat{\mathbf{s}}_{E, n+1 / 2}=\Delta \mathbf{s}_{E}-\left(\hat{\mathbf{s}}_{E, n+1 / 2}-\mathbf{s}_{E, n}\right)
$$

where, at this stage, $\left(\hat{\mathbf{s}}_{n+1 / 2}-\mathbf{s}_{n}\right)$ is a known quantity, we must solve:

$$
\Delta \mathbf{s}_{E}=\operatorname{Arg} \min _{\Delta \mathbf{s}_{E} \in \mathbf{A}_{\mathbf{d} E}^{\star}} e_{R C, E}^{2}\left(\Delta \mathbf{s}_{E}-\left(\hat{\mathbf{s}}_{E, n+1 / 2}-\mathbf{s}_{E, n}\right)\right)
$$

where the constitutive relation error is:

$$
e_{R C, E}^{2}\left(D \mathbf{s}_{E}\right)=\left\|D \dot{\mathbf{e}}_{E}-\mathbf{H} D \mathbf{f}_{E}\right\|_{\mathbf{H}, E}^{2}+\left\|D \underline{\dot{W}}_{E}+\mathbf{h} D \underline{F}_{E}\right\|_{\mathbf{h}, E}^{2}
$$

the corresponding norms are:

$$
\|\square\|_{\mathbf{H}, E}^{2}=\int_{[0, T] \times \Omega_{E}}\left(1-\frac{t}{T}\right) \square \circ \mathbf{H}^{-1} \square d \Omega d t
$$

and:

$$
\|\square\|_{\mathbf{h}, E}^{2}=\int_{[0, T] \times \partial \Omega_{E}}\left(1-\frac{t}{T}\right) \square \cdot \mathbf{h}^{-1} \square d S d t
$$

\subsubsection{Choice of admissible radial time-space functions}

The choice of the approximation presented here is an improvement over the version introduced in [36]. The starting point is the introduction as unknowns of the radial time-space approximations of the corrections related to the inelastic strain and to the additional internal variables:

$$
\begin{aligned}
& \Delta \varepsilon_{\mathrm{p} E}(t, \underline{M})=\sum_{k=1}^{p} a^{k}(t) \mathbf{E}_{\mathbf{p}}^{k}(\underline{M}) \\
& \Delta \mathbf{X}_{E}(t, \underline{M})=\sum_{k=1}^{p^{\prime}} b^{k}(t) \mathbf{D}^{k}(\underline{M})
\end{aligned}
$$

Using the E-admissibility conditions, one determines the other quantities of 
interest in terms of the previous unknowns:

$$
\begin{aligned}
\left(\Delta \varepsilon_{E}, \Delta \underline{W}_{E}\right)(t, \underline{M}) & =\sum_{k=1}^{p} a^{k}(t)\left(\mathbf{E}^{k}, \underline{Z}^{k}\right)(\underline{M}) \\
\left(\Delta \boldsymbol{\sigma}_{E}, \Delta \underline{F}_{E}\right)(t, \underline{M}) & =\sum_{k=1}^{p} a^{k}(t)\left(\mathbf{C}^{k}, \underline{G}^{k}\right)(\underline{M}) \\
\Delta \mathbf{Y}_{E}(t, \underline{M}) & =\sum_{k=1}^{p^{\prime}} b^{k}(t) \mathbf{R}^{k}(\underline{M})
\end{aligned}
$$

where the space functions are linked by the relations:

$$
\mathbf{E}^{k}=\mathbf{E}_{\mathbf{p}}^{k}+\mathbf{K}^{-1} \mathbf{C}^{k} \quad \text { and } \quad \mathbf{R}^{k}=\Lambda \mathbf{D}^{k}
$$

and the space operators are defined through standard finite element approximation over the space domain $\Omega_{E}$.

Compared to the previous version of the radial loading time-space approximation, we obtain the same quality of approximation with only half the number of time functions.

\subsubsection{Definition of the best approximation}

In order to solve (65), the idea is to seek minima alternatively with respect to time (which leads to a system of differential equations) and to space (which leads to a "spatial" problem). Since the construction of the space functions is by far the most expensive step of this process, it is advantageous to store and reuse these functions. Thus, the space functions constructed up to Iteration $n$ are reused systematically during Iteration $n+1$. Let us note that a reduced basis can be shared by several substructures if these substructures are similar.

\subsubsection{Practical resolution technique}

Let us assume that we are dealing with Iteration $n+1$ and that we have at our disposal a reduced basis made up of the space functions $\left\{\left(\mathbf{E}_{\mathbf{p}}^{k}, \mathbf{D}^{k}\right)\right\}_{k=1, \ldots, m}$ for the approximation of the corrections related to the inelastic strain $\Delta \varepsilon_{\mathrm{p} E}$ and to the additional internal variables $\Delta \mathbf{X}_{E}$. The space functions related to the other quantities $\Delta \varepsilon_{E}, \Delta \underline{W}_{E}, \Delta \boldsymbol{\sigma}_{E}$ and $\Delta \underline{F}_{E}$ are also considered to be known.

Step 1: use of the reduced basis. One introduces the approximation:

$$
\begin{aligned}
& \Delta \varepsilon_{\mathrm{p} E}(t, \underline{M})=\sum_{k=1}^{m} a^{k}(t) \mathbf{E}_{\mathbf{p}}^{k}(\underline{M}) \\
& \Delta \mathbf{X}_{E}(t, \underline{M})=\sum_{k=1}^{m} b^{k}(t) \mathbf{D}^{k}(\underline{M})
\end{aligned}
$$


into the constitutive relation error (66) where only the time functions are the unknowns. Thus:

$$
a^{k}(0)=b^{k}(0)=0, \quad k=1, \ldots, m
$$

These time functions verify a linear differential equation in time with conditions at $t=0$ and $t=T$, whose solution is obtained classically. This is generally a rather small system. If the value of the constitutive relation error is small enough, one stops the process and selects the approximation obtained. Otherwise, one proceeds to Step 2.

Step 2: adding new functions. One adds:

$$
\begin{aligned}
\Delta \varepsilon_{\mathrm{p} E}(t, \underline{M}) & =\sum_{k=1}^{m+r} a^{k}(t) \mathbf{E}_{\mathbf{p}}^{k}(\underline{M}) \\
\Delta \mathbf{X}_{E}(t, \underline{M}) & =\sum_{k=1}^{m+r} b^{k}(t) \mathbf{D}^{k}(\underline{M})
\end{aligned}
$$

where both $\left\{\left(a^{k}, b^{k}\right)\right\}_{k=m+1, \ldots, m+r}$ and $\left\{\left(\mathbf{E}_{\mathbf{p}}^{k}, \mathbf{D}^{k}\right)\right\}_{k=m+1, \ldots, m+r}$ are now unknowns. In practice, one takes $r=1$. One seeks a minimum alternatively over the time functions and the space functions. These subiterations begin with an initialization of the time functions. In order to do that, one uses the residue written in terms of the reference points and reference times. The minimization with respect to the space functions is standard, with twice the size of a classical finite element calculation. The minimization with respect to the time functions leads to a differential equation with conditions at $t=0$ and $t=T$, which can be easily solved using a standard technique. In practice, one stops after 1 or 2 subiterations. What is important is the complete calculation of the reduced spatial basis.

\subsection{Numerical example of the resolution of a micro problem}

In order to illustrate how this technique is used in the multiscale strategy, let us go back to the example described in Section 5.5 and use the radial timespace approximation to represent the unknowns of a problem similar to the micro problem associated with a Type-II substructure in which the loading consists of the distribution of the Lagrange multiplier $\underline{W}_{E}^{M}$ alone. For the sake of simplicity, we assume that this loading consists of only a normal force distribution $f(t)$ over the top surface of the substructure (see Figure 15).

Figure 16 shows the evolution of the constitutive relation error associated with the search direction with respect to the number of functions, using two techniques: the first technique consisted in systematically building new pairs of time/space functions; the second technique consisted in first reusing the basis 


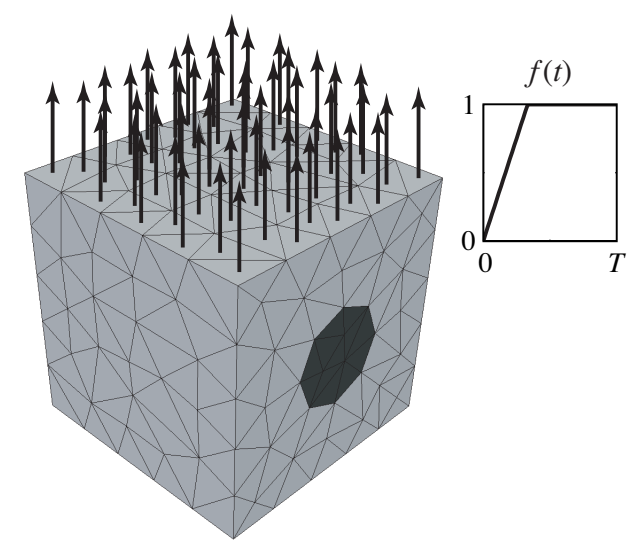

Fig. 15. Description of the micro problem and its loading

of space functions previously calculated to update the time functions alone, and only then seeking a new pair of time/space functions. One can see that the accuracy of the approximation is very good because the error was less than $1 \%$ using only 4 radial functions. However, one can observe that the convergence rate of the second technique is higher than that of the first. For example, in order to get less than $0.1 \%$ error, one needs to calculate 15 functions if one does not update the time functions, as opposed to only 8 functions with the updating procedure. Since the computing cost of the updating stage is much less than that of another space function, it is very important to update the time functions systematically.

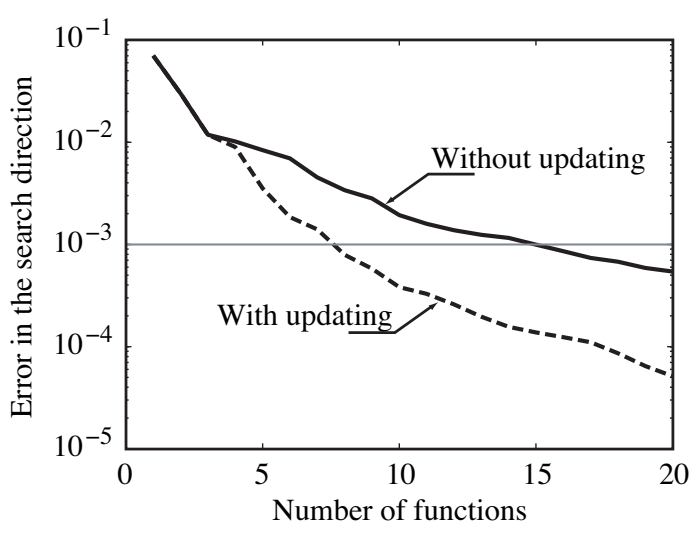

Fig. 16. Convergence of the approximation

Figure 17, shows the first four pairs, each constituted of one space function and one time function. The space functions are normalized and, thus, one can observe a decrease in the level of the corresponding time function. Figure 18 gives a comparison of the radial time-space approximation and the classical incremental solution in terms of Von Mises stresses over the space and time domains.

A very important point is that the basis of space functions is a priori specific to the problem and the loading for which it is defined, but it can be reused 


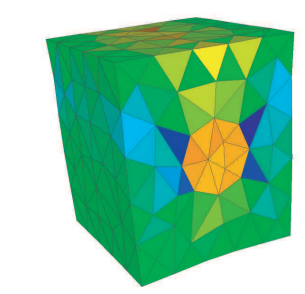

$1^{\text {st }}$ pair
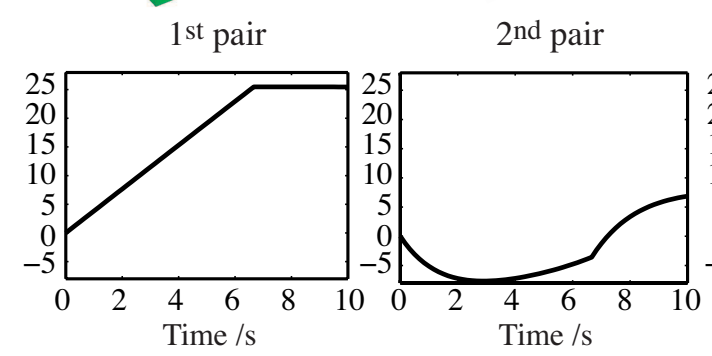
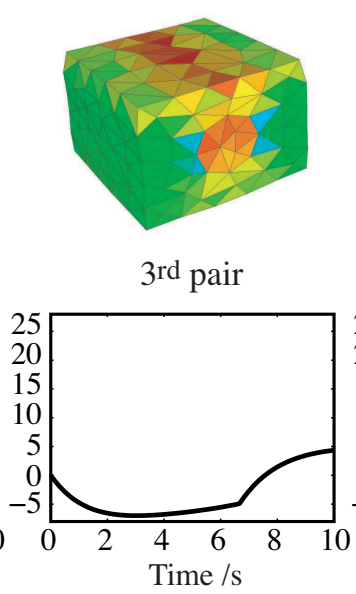
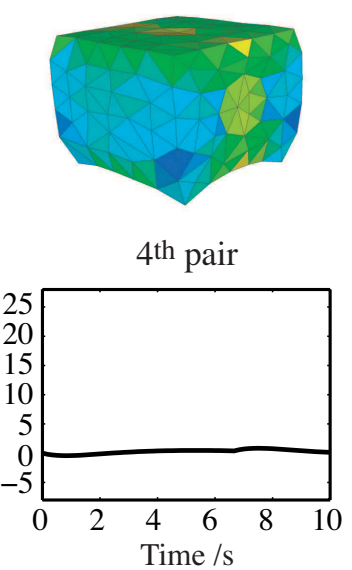

Fig. 17. The first four radial time-space functions for the problem

to solve another problem with comparable accuracy. For example, we solved the previous example with 6 functions and reused these functions for all the loading cases of Figure 19. In order to do that, we carried out a single update stage and evaluated the corresponding error.

Table 20 shows that by updating the time functions alone using the same space functions as for a previous problem $f(t)$ one obtains an approximate solution of the new problem $f_{i}(t)$ with an accuracy comparable to that of the first problem. The robustness of the radial time-space approximation makes it well-adapted to multiresolution. Thus, this approximation technique is quite suitable for the multiscale strategy, which involves the resolution of a set of micro problems at each iteration of the LATIN method. We can reuse the same basis for every iteration of these micro problems, and even consider using a common basis for the whole set of substructures.

The coupling of the multiscale time and space aspects of Section 4 with the new version of the radial time-space approximation of Section 6 is being developed. Nevertheless, examples of the capabilities of the method have already been given in $[36,38]$.

\section{Conclusions}

The first version of the multiscale computational strategy described here has been applied to several large-scale engineering problems involving multiple scales, such as the prediction of damping in space launcher joints [50] or the simulation of microcracking in composite materials. The radial time-space approximation leads to a drastic reduction in calculation and storage costs, especially with the new version described here, without affecting the robustness 


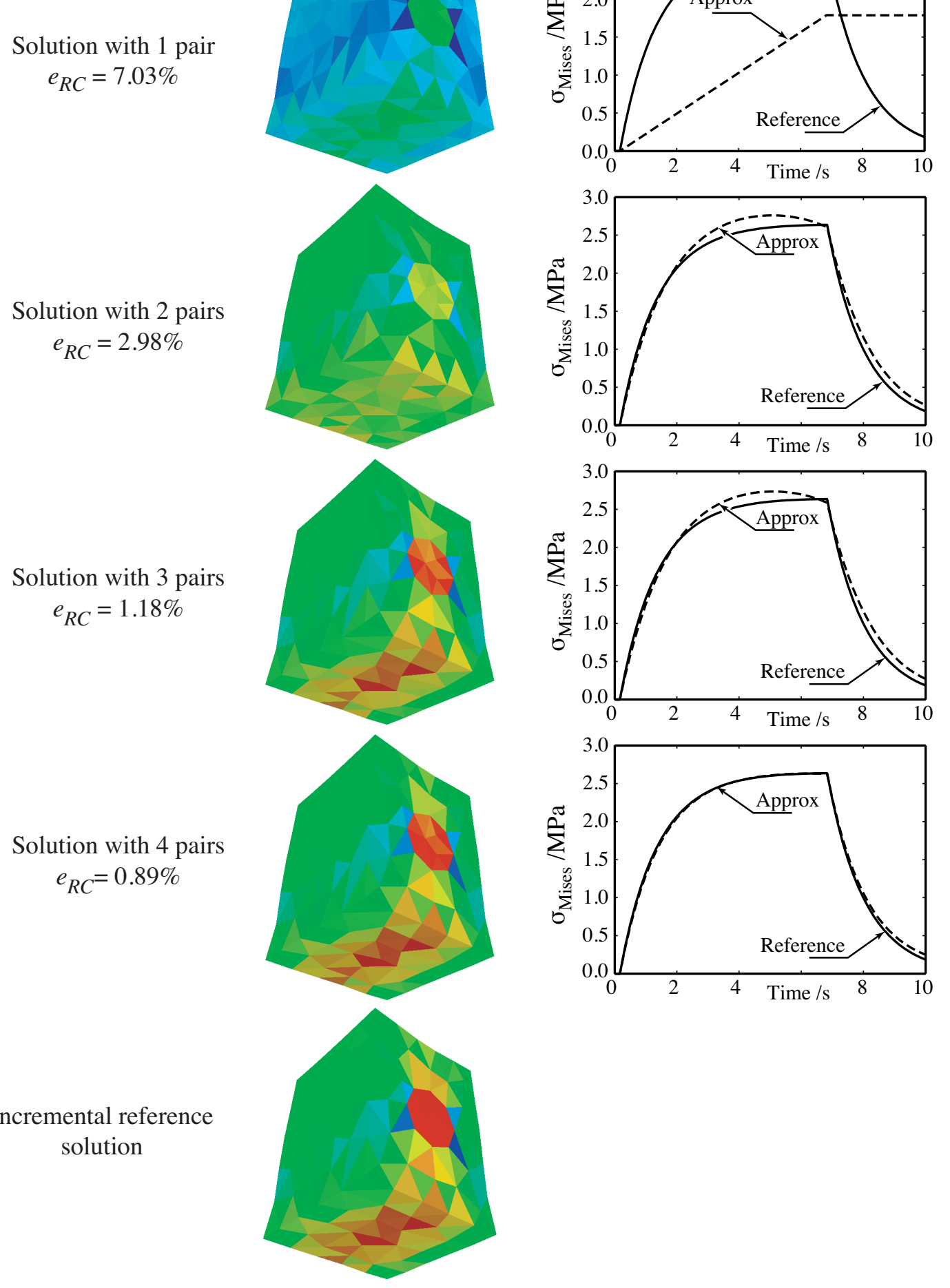

Fig. 18. Quality of the approximations with 1, 2, 3 and 4 pairs 


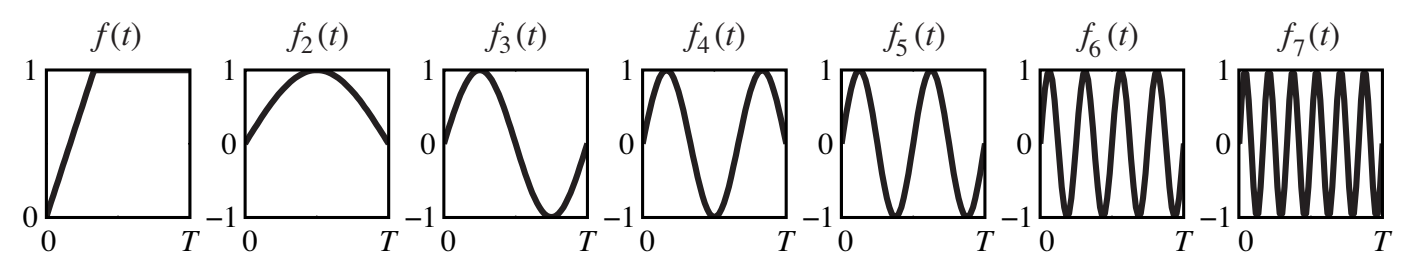

Fig. 19. The different loading cases for the micro problem

\begin{tabular}{|l||c||c|c|c|c|c|c|}
\hline Loading case & $f(t)$ & $f_{2}(t)$ & $f_{3}(t)$ & $f_{4}(t)$ & $f_{5}(t)$ & $f_{6}(t)$ & $f_{7}(t)$ \\
\hline Error $e_{C R}$ & $0.179 \%$ & $0.183 \%$ & $0.239 \%$ & $0.291 \%$ & $0.332 \%$ & $0.411 \%$ & $0.434 \%$ \\
\hline
\end{tabular}

Fig. 20. Reuse of a space function with the radial time-space approximation

and the effectiveness of the method. This, however applies only to quasi-static problems. A first improvement, currently in progress, consists in the introduction of a new algebra, i.e. a general mathematical framework, in which all functions are described thanks to the radial time-space approximation. Another work in progress is the extension of the scalability proven for multiple space scales to the general case. The final development, still under quasi-static conditions, will deal with the extension to large-displacement problems following the mathematical framework already proposed in [37].

\section{References}

[1] P. Cresta, O. Allix, C. Rey, S. Guinard, Comparison of multiscale nonlinear strategies for post-buckling analysis, Computer Methods in Applied Mechanics and Engineering 196 (8) (2007) 1436-1446.

[2] P.-A. Guidault, O. Allix, L. Champaney, J.-P. Navarro, A micro-macro approach for crack propagation with local enrichment, in: Proceedings of 7 th International Conference on Computational Structures Technology, CST 2004, Lisbon, Portugal, 7-10 September, 2004.

[3] P. Ladevèze, Multiscale modelling and computational strategies for composites, International Journal for Numerical Methods in Engineering 60 (2004) 223-253.

[4] P. Ladevèze, G. . Lubineau, D. Violeau, Computational damage micromodel of laminated composites, International Journal of Fracture 137 (1) (2006) 139-150.

[5] E. Sanchez-Palencia, Comportement local et macroscopique d'un type de milieux physiques hétérogènes, International Journal for Engineering Science 12 (1974) 231-251.

[6] E. Sanchez-Palencia, Non homogeneous media and vibration theory, Lecture Notes in Physics 127 (1980) Springer-Verlag. 
[7] F. Feyel, A multilevel finite element method $\left(F E^{2}\right)$ to describe the response of highly nonlinear structures, using generalized continua, Computer Methods in Applied Mechanics and Engineering 192 (2003) 3233-3244.

[8] F. Devries, H. Dumontet, G. Duvaut, F. Léné, Homogenization and damage for composite structures, International Journal for Numerical Methods in Engineering 27 (2) (1989) 285-298.

[9] T. Zohdi, J. Oden, G. Rodin, Hierarchical modeling of heterogeneous bodies, Computer Methods in Applied Mechanics and Engineering 138 (1-4) (1996) 273-298.

[10] J. Oden, K. Vemaganti, N. Moës, Hierarchical modeling of heterogeneous solids, Computer Methods in Applied Mechanics and Engineering 172 (1-4) (1999) 325.

[11] J. Fish, K. Shek, M. Pandheeradi, M. S. Shephard, Computational plasticity for composite structures based on mathematical homogenization: Theory and practice, Computer Methods in Applied Mechanics and Engineering 148 (1-2) (1997) 53-73.

[12] M. Lefik, B. Schrefler, Modelling of nonstationary heat conduction problems in micro-periodic composites using homogenisation theory with corrective terms, Archives of Mechanics 52 (2) (2000) 203-223.

[13] C. Huet, Application of variational concepts to size effects in elastic heterogeneous bodies, Journal of the Mechanics and Physics of Solids 38 (6) (1990) 813-841.

[14] V. Kouznetsova, M. Geers, W. Brekelmans, Multi-scale constitutive modelling of heterogeneous materials with a gradient-enhanced computational homogenization scheme, International Journal for Numerical Methods in Engineering 54 (2002) 1235-1260.

[15] T. Zohdi, P. Wriggers, Introduction to computational micromechanics, Springer Verlag, 2005.

[16] T. Hughes, Multiscale phenomena: Green's function, the dirichlet-to-neumann formulation, subgrid scale models, bubbles and the origin of stabilized methods, Computer Methods in Applied Mechanics and Engineering 127 (1995) 387-401.

[17] P. Ladevèze, O. Loiseau, D. Dureisseix, A micro-macro and parallel computational strategy for highly heterogeneous structures, International Journal for Numerical Methods in Engineering 52 (1-2) (2001) 121-138.

[18] A. Combescure, A. Gravouil, A numerical scheme to couple subdomains with different time-steps for predominantly linear transient analysis, Computer Methods in Applied Mechanics and Engineering 191 (11-12) (2002) 1129-1157.

[19] V. Faucher, A. Combescure, A time and space mortar method for coupling linear modal subdomains and non-linear subdomains in explict structural dynamics, Computer Methods in Applied Mechanics and Engineering 192 (2003) 509-533. 
[20] A. Gravouil, A. Combescure, Multi-time-step and two-scale domain decomposition method for non-linear structural dynamics, International Journal for Numerical Methods in Engineering 58 (2003) 1545-1569.

[21] A. Gravouil, A. Combescure, Multi-time-step explicit implicit method for nonlinear structural dynamics, International Journal for Numerical Methods in Engineering 50 (2001) 199-225.

[22] C. Farhat, M. Chandesris, Time-decomposed parallel time-integrators: theory and feasibility studies for fluid, structure, and fluid-structure applications, International Journal for Numerical Methods in Engineering 58 (2003) 13971434 .

[23] T. Belytschko, P. Smolinski, W. K. Liu, Stability of multi-time step partitioned integrators for first-order finite element systems, Computer Methods in Applied Mechanics and Engineering 49 (3) (1985) 281-297.

[24] C. L. Bottasso, Multiscale temporal integration, Computer Methods in Applied Mechanics and Engineering 191 (25-26) (2002) 2815-2830.

[25] D. Dureisseix, P. Ladevèze, D. Néron, B. A. Schrefler, A multi-time-scale strategy for multiphysics problems: application to poroelasticity, International Journal for Multiscale Computational Engineering 1 (4) (2003) 387-400.

[26] Y. Maday, G. Turinici, A parareal in time procedure for the control of partial differential equations, Comptes Rendus Académie des Sciences Paris I(335) (Issue 4) (2002) 387-392.

[27] M. Maitournam, B. Pommier, J.-J. Thomas, Determination de la reponse asymptotique d'une structure anelastique sous chargement thermomecanique cycliquedetermination of the asymptotic response of a structure under cyclic thermomechanical loading, Comptes Rendus Mecanique 330 (10) (2002) 703708 .

[28] F. Comte, H. Maitournam, P. Burry, N. T. M. Lan, A direct method for the solution of evolution problems, Comptes Rendus Mecanique 334 (5) (2006) 317322 .

[29] J. Fish, W.Chen, Uniformly valid multiple spatial-temporal scale modeling for wave propagation in heterogeneous media, Mechanics of Composite Materials and Structures 8 (2001) 81-99.

[30] Q. Yu, J. Fish, Multiscale asymptotic homogenization for multiphysics problems with multiple spatial and temporal scales: a coupled thermo-viscoelastic example problem, International Journal of Solids and Structures 39 (2002) 6429-6452.

[31] T. Guennouni, On a computational method for cycling loading: the time homogenization, Mathematical Modelling and Numerical Analysis (in french) 22 (3) (1988) 417-455. 
[32] S. Akel, Q. S. Nguyen, Determination of the limit response in cyclic plasticity, in: Proceedings of 2nd International Conference on Computational Plasticity, Vol. 639-650, Barcelone, Spain, 1989.

[33] P. Ladevèze, P. Rougée, (Visco)plasticity under cyclic loadings: properties of the homogenized cycle, Comptes Rendus Académie des Sciences Paris (in french) II(301) (13) (1985) 891-894.

[34] P. Ladevèze, New advances in the large time increment method, in: P. Ladevèze, O. C. Zienkiewicz (Eds.), New advances in computational structural mechanics, Elsevier, 1991, pp. 3-21.

[35] J.-Y. Cognard, P. Ladevèze, A large time increment approach for cyclic plasticity, International Journal of Plasticity 9 (1993) 114-157.

[36] P. Ladevèze, A. Nouy, On a multiscale computational strategy with time and space homogenization for structural mechanics, Computer Methods in Applied Mechanics and Engineering 192 (2003) 3061-3087.

[37] P. Ladevèze, Nonlinear computational structural mechanics - new approaches and non-incremental methods of calculation, Springer Verlag, 1999.

[38] A. Nouy, P. Ladevèze, Multiscale computational strategy with time and space homogenization: a radial-type approximation technique for solving microproblems, International Journal for Multiscale Computational Engineering 2 (4) (2004) 557-574.

[39] P.-A. Boucard, P. Ladevèze, H. Lemoussu, A modular approach to 3-D impact computation with frictional contact, Computer and Structures 78 (1-3) (2000) $45-52$.

[40] K. Eriksson, C. Johnson, V. Thomée, Time discretization of parabolic problems by the discontinuous galerkin formulation, RAIRO Modélisation Mathématique et Analyse Numérique 19 (1985) 611-643.

[41] P. Ladevèze, A. Nouy, O. Loiseau, A multiscale computational approach for contact problems, Computer Methods in Applied Mechanics and Engineering 191 (2002) 4869-4891.

[42] P. Ladevèze, J.-P. Pelle, Mastering calculations in linear and nonlinear mechanics, Springer NY, 2004.

[43] P. Ladevèze, New algorithms: mechanical framework and development (in french), Tech. Rep. 57, LMT-Cachan (1985).

[44] D. Ryckelynck, L. Hermanns, F. Chinesta, E. Alarcon, An efficient 'a priori' model reduction for boundary element models, Engineering Analysis with Boundary Elements 29 (8) (2005) 796-801.

[45] A. Ammar, B. Mokdad, F. Chinesta, R. Keunings, A new family of solvers for some classes of multidimensional partial differential equations encountered in kinetic theory modelling of complex fluids, Journal of Non-Newtonian Fluid Mechanics 139 (3) (2006) 153-176. 
[46] A. Ammar, B. Mokdad, F. Chinesta, R. Keunings, A new family of solvers for some classes of multidimensional partial differential equations encountered in kinetic theory modelling of complex fluids: Part ii: Transient simulation using space-time separated representations, Journal of Non-Newtonian Fluid Mechanics 144 (2-3) (2007) 98-121.

[47] A. Nouy, A generalized spectral decomposition technique to solve a class of linear stochastic partial differential equations, Computer Methods in Applied Mechanics and Engineering 196 (45-48) (2007) 4521-4537.

[48] A. Chatterjee, An introduction to the proper orthogonal decomposition, Current Science 78 (7) (2000) 808-817.

[49] P. Ladevèze, A computational technique for the integrals over the time-space domain in connection with the LATIN method, Tech. Rep. 193, LMT-Cachan (1997).

[50] A. Caignot, P. Ladevèze, D. Néron, S. Le Loch, V. Le Gallo, K. Ma, T. Romeuf, Prediction of damping in space lauch vehicles using a virtual testing strategy, in: Proc. of 6th International Symposium on Launcher Technologies, 2005.

[51] F. Brezzi, L. Marini, A three-field domain decomposition method, in: Proceedings of the 6th International Conference on Domain Decomposition Methods, 1993, pp. 27-34.

[52] J.-L. Lions, Y. Maday, G. Turinici, Résolution d'edp par un schéma en temps "pararéel", Comptes-Rendus de l'Académie des Sciences I(332) (2001) 661-668.

[53] F. Feyel, J.-L. Chaboche, $F E^{2}$ multiscale approach for modelling the elastoviscoplastic behaviour of long fibre sic/ti composite materials, Computer Methods in Applied Mechanics and Engineering 183 (3-4) (2000) 309-330.

[54] P. Ladevèze, D. Néron, P. Gosselet, On a mixed and multiscale domain decomposition method, Computer Methods in Applied Mechanics and Engineering 196 (2007) 1525-1540.

[55] L. Séries, F. Feyel, F.-X. Roux, A domain decomposition method with two Lagrange multipliers, in: Proceedings of the 16th congrès français de mécanique, 2003 , in french.

[56] P. Gosselet, C. Rey, On a selective reuse of krylov subspaces in newton-krylov approaches for nonlinear elasticity, in: Proceedings of the 14th conference on domain decomposition methods, 2002, pp. 419-426.

[57] P. Gosselet, C. Rey, Non-overlapping domain decomposition methods in structural mechanics, Archives of Computational Methods in Engineering (2005) submitted.

[58] G. Rebel, K. Park, C. Felippa, A contact formulation based on localized lagrange multipliers: formulation and application to two dimensional problems, International Journal for Numerical Methods in Engineering 54 (2002) 263-297. 
[59] P. Le Tallec, Y.-H. De Roeck, M. Vidrascu, Domain-decomposition methods for large linearly elliptic three dimensional problems, Journal of Computational and Applied Mathematics 34 (1991) 93-117.

[60] C. Farhat, M. Lesoinne, P. Le Tallec, K. Pierson, D. Rixen, FETI-DP: a dualprimal unified FETI method - part i: a faster alternative to the two-level FETI method, International Journal for Numerical Methods in Engineering 50 (7) (2001) 1523-1544. 